Portland State University

PDXScholar

9-15-1995

\title{
Use of Language Learning Strategies by Proficient and Less Proficient Learners
}

Charlene Frances Mills

Portland State University

Follow this and additional works at: https://pdxscholar.library.pdx.edu/open_access_etds

Part of the Bilingual, Multilingual, and Multicultural Education Commons Let us know how access to this document benefits you.

\section{Recommended Citation}

Mills, Charlene Frances, "Use of Language Learning Strategies by Proficient and Less Proficient Learners" (1995). Dissertations and Theses. Paper 5045.

https://doi.org/10.15760/etd.6921

This Thesis is brought to you for free and open access. It has been accepted for inclusion in Dissertations and Theses by an authorized administrator of PDXScholar. Please contact us if we can make this document more accessible: pdxscholar@pdx.edu. 


\section{THESIS APPROVAL}

The abstract and thesis of Charlene Frances Mills for the Master of Arts in TESOL were presented on September 15, 1995, and accepted by the thesis committee and the department.

COMMITTEE APPROVALS:

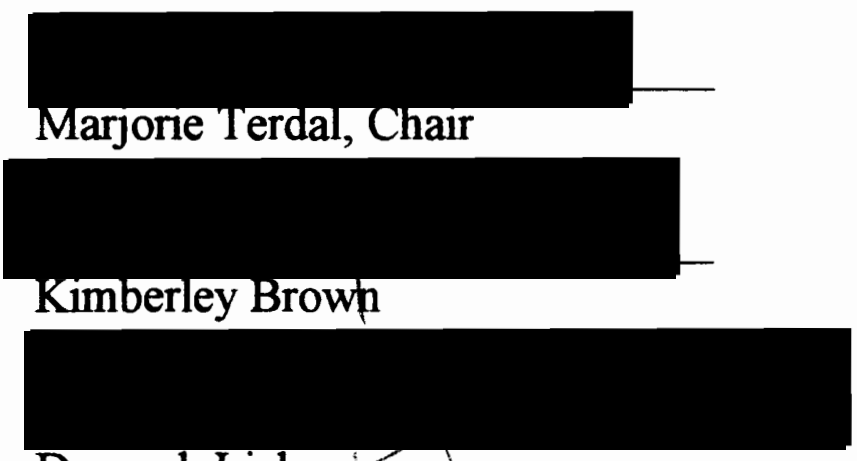

Devorah Lieberman

Representative of the Office of

Graduate Studies

DEPARTMENT APPROVAL:

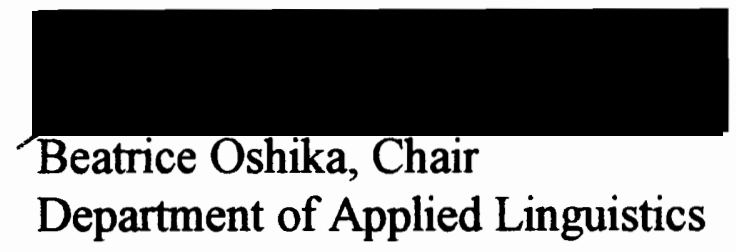

$* * * * * * * * * * * * * * * * * * * * * * * * * * * * * * * * * * * * * * * * * * * * * * * * * * * * * * * * * * * * * * * * *$

ACCEPTED FOR PORTLAND STATE UNIVERSITY BY THE LIBRARY

by on 20 Pewember 1985 


\begin{abstract}
An abstract of the thesis of Charlene Frances Mills for the Master of Arts in TESOL presented September 15, 1995.
\end{abstract}

Title: Use of Language Learning Strategies by Proficient and Less Proficient Learners

Recent research has found that the use of good language learning strategies can affect students' chances of gaining proficiency in a second language.

The purpose of this study was to see if there is a relationship between strategy use and language learning proficiency. It sought to answer these questions: (1) Does a successful learner use different strategies on specific tasks than does a less successful learner? and (2) Will the successful learner use more metacognitive and affective strategies while doing tasks than will a less successful learner?

For the first part of the study, 17 students in an ESL program at an urban university in the northwest were selected. Using the results from a self-report survey, the Strategy Inventory for Language Learning (SILL), and the students' scores from standardized examinations, these students were divided into two groups, a proficient group and a less proficient group. For the second part of the study, two subjects from the first part (one proficient and one less proficient) were selected to participate in a Think-Aloud protocol as they completed three tasks. The objective was to 
see if these two students used different strategies as they completed specific tasks and if the proficient learner used more metacognitive and affective strategies that the less proficient learner.

Mean scores were computed for the subjects on the first part of the study. Two-tailed probability tests were computed to determine if differences existed between the proficient and less proficient group. A significant difference was found between the two groups strategies from the memory strategy group. Analysis of the second part of the study revealed that the successful student used more of the appropriate strategies on two out of three of the tasks than did the less proficient learner and only slightly less of the appropriate strategy on the other task. The successful learner employed more metacognitive strategies on two out of three of the tasks and she used more of these strategies overall. The successful learner also used more affective strategies on all tasks.

The pracitical benefit of the study is that student awareness of good strategy use, tailored to specific tasks could lead to improved second language acquisition. 


\title{
USE OF LANGUAGE LEARNING STRATEGIES BY PROFICIENT AND LESS PROFICIENT LEARNERS
}

\section{by}

CHARLENE FRANCES MILLS

A thesis submitted in partial fulfillment of the requirements for the degree of

\author{
MASTER OF ARTS \\ in \\ TESOL
}

Portland State University

1995 


\section{DEDICATION}

I would like to dedicate this thesis to my son, Jeffrey, who has always believed in me and supported me in everything I have ever done. Without him, my life would be infinitely less full. 


\section{ACKNOWLEDGEMENTS}

I would like to acknowledge the following members of my thesis committee for their support: Marjorie Terdal, Kimberley Brown, and Devorah Lieberman. I would especially like to recognize my thesis advisor, Marjorie Terdal, for her constructive ideas and all the time she devoted to editing my work and offering many valuable suggestions. It is through her constant dedication to excellence that many students in the TESOL program are able to produce theses which satisfy the high standards of the Applied Linguistics Department at this university. 


\section{TABLE OF CONTENTS}

CHAPTER

PAGE

ACKNOWLEDGEMENTS...................................... i

LIST OF TABLES................................................. iv

LIST OF FIGURES.............................................

I INTRODUCTION.............................................. 1

Research Questions....................................... 5

Method .................................................... 7

Potential Benefits of Study............................... 9

Glossary of Terms.......................................... 10 .

II REVIEW OF THE LITERATURE............................. 13

Learning Strategies....................................... 14

Strategy Measurements..................................... 27

Learner Variables......................................... $\quad 35$

Successful/Unsuccessful Learners...................... 47

Summary ................................................. 50

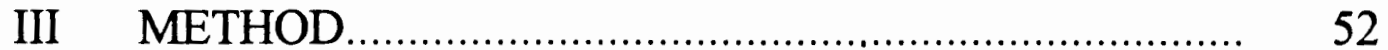

Subjects and Sampling Procedures..................... 54

SILL Survey Study Procedures.......................... 55

Think-Aloud Protocol (TAP) Procedures.............. $\quad 58$

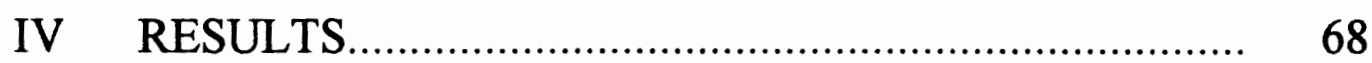

Research Question One.................................... 68

Research Question Two.................................... 71 
Research Question Three................................ 72

Research Question Four................................... 73

Research Question Five.................................. $\quad 77$

Overall Strategy Use........................................ 79

Summary........................................................ 81

V DISCUSSION AND CONCLUSION

Discussion................................................... 82

Conclusion...................................................... 93

REFERENCES ...................................................... 106 APPENDICES

A. SILL SURVEY ................................................ 114

B. DEFINITIONS OF LANGUAGE LEARNING STRATEGIES USED IN SILL ............................ 123

C. THINK-ALOUD PROTOCOL TASKS.................... 139

D. SAMPLES OF TRANSCRIPTS FROM THINKALOUD PROTOCOL ........................................ 144

E. STATISTICS FROM SILL................................ 148

F. STUDENT DATA FROM SILL AND THINKALOUD PROTOCOL ........................................ 155 


\section{LIST OF TABLES}

TABLE

PAGE

I Summary of Statistics From SILL ..................... 67

II Strategy Use in Think-Aloud Tasks................... 72

III Proficiency Ranking of Proficient Learners.......... 84 


\section{LIST OF FIGURES}

FIGURE

PAGE

1. Group means of reported strategy use for the two groups, proficient and less proficient learners, in the six categories of the SILL survey and the means of the overall SILL averages for the two groups..................... 70

2. Total number of strategies used by the two subjects in Think-Aloud Protocol for each of the six category groups.

3 Number of strategies used by the two subjects during each Think-Aloud Protocol task and for the three tasks combined. 


\section{CHAPTER I}

\section{INTRODUCTION}

The first part of the present study attempted to identify language learning strategies (LLSs) that are used by learners at different proficiency levels. Specifically, it looked at the strategies the most and least proficient learners in a group reported using to see if there were statistically significant differences between the two. The second part of the study delved deeper into LLS differences among learners by selecting for further investigation two members of the sample with similar strategy-use profiles, one evaluated as a proficient learner and one a less proficient learner. Using a Think Aloud Protocol the students were asked to reveal their feelings and think aloud as they went through a series of tasks. The objective of this part of the study was to see if results of these activities can help researchers better understand the relationship between strategy use on specific tasks and language proficiency. Additionally, it could increase awareness of the different strategy combinations used on specific tasks by a proficient and a less proficient learner. Finally, the results of the two parts of the study could help researchers see if there is a difference between what strategies learners reported they used on the survey and what they actually used on the set of tasks. This part of the study was limited only two subjects because of the time intensive nature of training the subjects, having them do the think-aloud activities and collecting and 
analyzing the data. Other researchers (Vann \& Abraham, 1990; Ericsson \& Simon, 1990; Perl, 1979) have used only two subjects or small numbers of subjects in their think-aloud studies

While research on learning strategies in other fields has been going on for many years, it is only within the last decade that researchers in second language acquisition (SLA) have begun to seriously investigate the strategies students use when they attempt to learn a second language. Rubin (1975), in one of the earliest articles, found in her research that good language learners had the same characteristics as good learners in other fields: willing and accurate guessing, a strong drive to communicate, willingness to take risks, attention to form and meaning, and monitoring learning. She reasoned that, if researchers could discover how strategy choice influenced SLA, this information could be used to help less successful students improve their chances of gaining language proficiency. Teachers could promote strategy use in the classroom by making students aware of the kinds of strategies available to them and training students in the use of strategies. Once mastered in the classroom setting, these would be available to them in self-directed learning both in and out of the classroom.

If strategy use does help in SLA, then two important questions that might be asked are: (1) how does strategy use differ between successful and less successful students, and (2) why do students choose the strategies they use? While most of the research has shown that successful learners use more strategies and use them more frequently, Oxford (1990b) 
reported that some studies have come to the conclusion that many learners use a wide range of strategies. Although even unsuccessful learners are familiar with and use many different strategies, the difference seems to be between how successful and unsuccessful learners use them. This is supported in research by Vann and Abraham (1990), where two unsuccessful students were studied. Each used a variety of strategies on different tasks. The problem, according to these researchers, seemed to be that the subjects did not choose the strategies that were best suited for particular tasks. Also in Perl's studies (1979) unsuccessful learners consistently used strategies on specific tasks that were different than less proficient learners.

The question of why students choose specific strategies has been addressed in research by looking at learner variables. A few that have shown relationships with strategies are anxiety, gender, culture, and personality type. Strategies that manage the emotions and reduce anxiety have been shown to be related to students' self -esteem and their willingness to take risks (Bacon \& Finnemann, 1990; Ehrman, 1994; Ehrman \& Oxford, 1995). Females tend to use more strategies (Ehrman \& Oxford, 1990). A study of university students (Nyikos, 1990) using colors and pictures to memorize vocabulary revealed that females did better than males when color association alone was used, while males outscored females when a combination of pictures and color associations were used. Reid (1987) found culture preferences for certain learning styles, with Korean, Arabic and Chinese students preferring visual learning and native 
speakers of English showing the least preference for tactile learning experiences. Students' personality type (whether they are extroverts or introverts, take in information by looking at the 'big picture' or linearly, process information objectively or by considering the situation, and prefer reaching conclusions or are open-ended) has been found to influence strategy choice (Ehrman \& Oxford, 1995, 1990, 1987). These researchers found that extroverts use more affective and visual strategies, students who prefer to look at the problem as a whole use more searching and communicating strategies, and those who have a preference for problem solving and take into account the situation as they make decisions use more general study strategies.

To collect data for their studies researchers have used different methods, including self-report instruments like questionnaires, interviews, diaries, and surveys. They also use instruments like the Think-Aloud Protocol, where students are asked to think out loud as they do tasks so that the researcher can capture the strategies that students are using immediately. One of the more popular self-report surveys is Oxford's (1990) Strategy Inventory for Language Learning (SILL). This instrument, which divides strategies into six major groups: memory, cognitive, compensation, metacognitive, affective, and social, has allowed researchers to collect data from many different large groups and do statistical comparisons to test for validity across studies. Oxford, Ehrman, and other researchers have used it extensively in their research. The studies of Oxford, Ehrman and Nyikos referred to above all used this 
instrument.

Oxford and Crookall (1989), reported finding that what strategies students reported using did not always correspond with what they actually used in tutorial sessions. The value of doing think-aloud research is that these differences can be investigated. The Think-Aloud Protocol was first developed in the 1920's by researchers in cognitive psychology for use in first language acquisition (Ericsson \& Simon, 1993). It has been recently introduced into SLA because it is believed by some researchers that human information processing for SLA is the same as for other learning activities (Dechert, 1987; Rubin, 1987, O'Malley \& Chamot, 1985). These are the two data collection instruments that were used in this study.

As more connections between learner variables and strategies are revealed and more evidence is collected that leads to understanding about how successful and unsuccessful learners use strategies differently, the possibility for applying this information in the classroom to educate students in effective strategy use increases. Armed with knowledge about (1) the kinds of strategies that work best with specific tasks, and (2) the kinds of learner variables that sometimes lead students to make wrong strategy choices, students can adjust their behavior and increase their potential for language learning success both inside and outside of the classroom.

\section{Research Questions}

The first part of the study used the SILL to collect data on what 
strategies students reported using and to see if a relationship existed between proficiency and: (1) level of strategy use, and (2) level of metacognitive and affective strategies used by proficient and less proficient learners. The second part of the study used a Think-Aloud Protocol to discover the difference between the specific strategies used by a proficient learner and a less proficient learner as they completed a series of tasks.

Through investigation of these relationships the study sought to answer the following questions.

Research question one.

Do proficient learners use more overall strategies, as measured by the SILL, when trying to learn a second language than less proficient learners? The six categories of strategies are: (1) memory - applying techniques to learning a language that help them remember, (2) cognitive using one's mental processes, (3) compensation - counterbalancing for missing knowledge, (4) metacognition - organizing and managing learning, (5) affective - managing emotions, and (6) social - learning with others. In all six of these strategy groups do proficient learners score three or higher on a five point scale and non proficient learners score less than three? To score three on the use of a specific strategy students are saying they use that strategy about half the time.

Research question two.

Do proficient learners use more metacognitive and affective strategies than do less proficient learners, as measured by the SILL? 
Research question three.

Is the difference in the number of metacognitive and affective strategies as measured by the SILL used by proficient learners and less proficient learners greater than the difference in the number of other strategies they use: memory, cognitive, compensation, and social?

Research question four.

Do proficient learners use combinations of strategies for specific tasks that are different than those used by less proficient learners on the same tasks, as measured by a Think-Aloud Protocol?

Research question five.

While learners are in the process of doing specific tasks will proficient learners use more metacognitive and affective strategies than will less proficient learners, as measured by the Think-Aloud Protocol?

Method

Measurement instruments and sample selection.

The measure of student proficiency was drawn from: (1) the retired non-secure version of the Michigan Test of English Language Proficiency (MTELP) and (2) the listening portion of the Comprehensive English Language Test (CELT). The instrument used in the first part of the study to measure language students' learning strategies was the SILL. The instrument used in the second part of the study to collect information on actual strategies used during the completion of specific tasks was the Think-Aloud Protocol. 
The students who participated in the first part of the research, were those students in the upper intermediate and advanced levels of the reading classes in the ESL program at an urban university in the northwest. Two students from the upper intermediate class, one proficient and one less proficient, were selected for the second part of the study from those participating in the first part of the study by asking for volunteers.

\section{Data collection and analysis.}

The students in the first part of the study were divided into two groups, proficient students and less proficient students, on the basis of their scores on the MTELP and the CELT. The SILL profiles of the students were statistically compared with students' proficiency measurement to examine the differences in strategy use for the two groups.

The students in the second part of the study participated in a series of three tasks that were monitored by the researcher. To determine what strategies the students were engaging in while doing the tasks, a ThinkAloud Protocol was used, whereby the students related verbally what they were thinking as they went through the tasks. The results of the tests and the researcher's observations were used to analyze the relationships between: (a) tasks and strategy choice, (b) different strategies used by the two subjects, and (c) actual strategies used and strategy use reported on the SILL. By comparing the results of the SILL and the think-aloud data, the researcher was able to identify the differences between what strategies 
these two students reported that they used and what they actually used when completing specific tasks.

\section{Potential Benefits of the Study}

Current research in the field of second language acquisition is trying to establish a connection between the kinds of learning strategies that learners use and their language learning success (Vann \& Abraham, 1990; Ehrman \& Oxford, 1995; Oxford, 1993; Rubin, 1975). This study provides some added insight into the relationships between second language learners' proficiency and the learning strategies they choose. It also reveals which strategies are used for specific tasks by a proficient learner and which are used by a less proficient learner. By improving our knowledge about these differences, teachers and trainers can make students more aware of the strategies they use and which ones are more likely to help them succeed at different tasks. The reason it is important to investigate this topic is that, if teachers can help students to develop awareness of the strategies they are using and to be more careful and conscious in their strategy choice, their efforts toward second language proficiency may become more successful. 


\section{Glossary of Terms}

Comprehensive English Language Test (CELT), listening portion: The CELT covers three sections: listening structure and vocabulary. Only the listening portion was used in this study. It is $\mathbf{4 0}$ minutes long and includes 50 oral questions. The first group of 20 are mainly who, what, when, and where questions. The second group of twenty items requires that the listeners select appropriate paraphrases out of four possibilities. For the last 10 items the listeners are required to listen to dialogues and then answer questions about them (Madsen, 1990).

\section{Long Term Memory (LTM)/ Short Term Memory (STM): Cognitive} psychologists describe the way we learn in terms of STM and LTM. Information initially enters STM, which has a limited capacity. For it to become knowledge it must be transferred into LTM. When new information is taken in, one can retrieve information from LTM to make relationships with it. The relevance to this study is that the Think-Aloud Protocol captures information on learning strategies while it is still in short term memory (and unedited), whereas the SILL survey and other selfreports (diaries, questionnaires, interviews) rely on students recalling from LTM what strategies they think they use. STM information, in this situation, should provide a more accurate representation of the strategies actually being used by the students. 
Michigan Test of English Language Proficiency (MTELP): The MTELP is a retired form of the Michigan placement battery of tests. It is a multiple choice objective test of grammar, vocabulary and reading comprehension. The first two portions include 40 items and the last portion includes 20 items (Madsen, 1990).

Proficient Learner/Less Proficient Learner: In this study both proficient learners and less proficient learners were drawn from the upper intermediate or advanced class of the ESL program at an urban university in the northwest. Both had taken the retired unsecure version of the Michigan Test for Language Proficiency and the listening portion of the Comprehensive English Language Test (CELT) near the end of spring term 1995. The grades from these two tests were weighted and combined, with $2 / 3$ weight for the Michigan test and 1/3 weight for the CELT. These were the weightings which the coordinator of the ESL program suggested. A proficient learner had a weighted average score of $70 \%$ or over. A less proficient learner had a weighted average score of $66.6 \%$ or less. The groupings were made in this way because there was a clustering of scores between $70 \%$ and $82 \%$, then a drop of almost four points to $66.6 \%$, with the final five students ranging from $66.6 \%$ to $57.4 \%$.

Strategy Inventory for Language Learning (SILL): The SILL is a self report survey with 80 questions, whereby second language students answer questions on the kinds of learning strategies they use while trying 
to learn and use a second language. The version used in this study was adopted from the original SILL to be used for non-native speakers of English. It is a fifty-item test that makes statements such as "I try to relax whenever I feel afraid of using English." The student is given five choices to select from, from 'always to almost always true of me' to 'never or almost never true of me'. The students answer questions about six different category groups: memory, cognitive, comprehension, metacognitive, affective, and social. (Oxford, 1990).

Think-Aloud Protocol (TAP): A procedure used by researchers who are trying to determine what kinds of strategies subjects are using as they work through a task. Students are required to think out loud as they complete an activity. They are not supposed to describe their actions, but just say aloud those thoughts that come into their head as they struggle with the task, as if they were in a room by themselves speaking their thoughts out loud. These thoughts are captured by audiotape and/or video tape, to be later analyzed by the researcher, whose goal it is to interpret from these thoughts what learning strategies the subjects were using. 


\section{CHAPTER II}

\section{REVIEW OF THE LITERATURE}

In what Kumaravadivelu (1994) refers to as the 'postmethod condition,' researchers have started to look beyond finding the magic method that will allow students of second languages to be successful in their pursuit of second language acquisition (SLA). Larsen-Freeman, in her deliberation on what helps second language (L2) learners succeed, states, "The question of differential success is one of the major conundrums of SLA (second language acquisition): Why is it that all individuals with normal faculties successfully acquire their first language but meet with different degrees of success when they attempt to master an L2?" (1991, p. 328). One of the areas that may contribute to the answer to that question and that has drawn a lot of attention in the past decade is language learning strategies (LLSs). An article on case study research (Oxford, Crookall, Cohen, Lavine, Nyikos \& Sutter, 1990) discusses the fact that research on strategies in most other fields has been going on for many years. Researchers in these other fields have found that "...the use of well chosen strategies typically distinguishes experts from novices; when compared with novices, experts use more strategies and more effective ones, to improve comprehension, retention and problems solving" (p. 198). Bialystok (1981) suggests that the pragmatic advantage for exploring learning strategies is that they can be taught to all L2 learners. 
With better command of appropriate strategies any learner has an increased opportunity to gain skills in a second language. If learning strategy use in other disciplines does have an impact on learning activities it is reasonable to expect that use of strategies in second language learning may enhance proficiency. Of course, we all use some sort of strategy or set of strategies when we approach any activity. The problem, then, is to look more closely at the types of strategies different kinds of learners use.

This review of the literature will look at the questions surrounding learning strategies: how they are defined and classified, why they are important to understand and study, and how second language learners apply different learning strategies as they endeavor to acquire a second language. Within this context, it will identify the types of strategies that are available to learners, look at a theory that provides a foundation for research, and review the different variables that are associated with strategy choice. It will also show how different groups of strategies and combinations of these groups may be favored by different learners. Finally, it will look at what research has shown are the differences between successful and unsuccessful learners.

\section{Learning Strategies}

Learning strategies can be defined in different ways, but they at least include techniques that help second language (L2) learners control their behavior and modify their actions in such ways that allow for them 
to more successfully complete our learning activities. They include what Oxford and Crookall (1989) refer to as learning-to-learn problem solving or study skills. They are the techniques that students of second languages use when they are going about learning a language.

The idea that second language learning could be facilitated by special learning techniques is fairly recent. Rubin, in one of the earliest articles (1975) that investigated learning strategies of second language learners, suggested that good language learners might be more successful because they used different techniques to acquire proficiency. Through observing students and talking to good language learners she was able to identify some of the characteristics of the good learners that led to certain strategy use: (1) willing and accurate guessers (efficiently gather and store information), (2) strong drive to communicate (use circumlocution and cognates), (3) lack of inhibitions (are willing to take risks), (4) attention to form (look for language patterns), (5) practice (seek opportunities to use language), (6) speech monitoring of self and others (evaluate performance), and (7) attention to meaning (pay attention to discourse rules and cultural clues).

The importance of her ideas was that they were in direct conflict with the popular notions of the day that some people had an inherent ability in SLA. What Rubin was suggesting was that if the strategies of good learners could be identified they might be taught to less successful learners. The theoretical basis for much of the recent interest in LLSs is summarized in Rubin (1987), who identifies ten reasons researchers feel 
that additional knowledge in strategies could lead to improvements in L2 proficiency: "(1) some language learners are more successful than others..., (2) the learning process includes both explicit and implicit knowledge..., (3) consciousness-raising is not incidental to learning..., (4) successful strategies can be used to good effect by less successful learners..., (5) teachers can promote strategy use..., (6) once trained, students become the best judge of how to approach the learning task..., (7) self-direction promotes learning both inside and outside the classroom...; (8) language learning is like other kinds of learning..., (9) the success of learner training in other subjects is applicable to language learning...; and (10) the critical faculty used by all humans in communicating is important in language learning" (pp. 15-18).

\section{A theoretical basis for learning strategies}

In 1985 O'Malley and Chamot noted that even with all the interest in learning strategies there had been no serious attempt to ground learning strategies in a theory that could show how they differ from other types of activity. They believed that the key to effective strategy use was the ability to build relationships between what is already known and the new information (schema theory). They first looked at a generative theory of SLA acquisition (Wittrock, Marks \& Doctorow, 1975) that based comprehension not on the sum of semantic, phonetic and episodic characteristics of sentences, but on identifying these with previous experiences stored in long term memory (LTM). These representations 
constructed from prior memory produce the psychological meaning of sentences. Wittrock (1983) proposed that the three components to this model are: (1) generation, (2) motivation, and (3) attention. Generation is the central process which can be explained by two parts, text and experience. Building relations between text, knowledge, and experience brings meaning to sentences. Motivation means that for comprehension to occur, there needs to be a willingness to invest effort and attribute success or failure to one's effort. Two components are involved in attention: the learner's short term orienting response to the sentence, and (2) the learners' long term voluntary response. If strategies promote these kinds of activities they should promote learning.

In order to establish a theoretical basis for applying learning strategies to research and use in SLA, O'Malley and Chamot adopted and elaborated on the information-processing theoretical model of the cognitive psychologists, particularly that of J. R. Anderson, whose ideas they cite extensively in their 1990 work. This model concentrates on two major functions: (1) metacognition, the management function, and (2) cognition, the processing function. Metacognition refers to knowledge about cognition or the regulation of cognition, where knowledge can apply to the learner's thoughts of his/her own cognition or that of others. Regulation involves regulation of cognition through planning, monitoring, and evaluating learning activities (Brown \& Palinscar, 1982). Cognitive psychology also identifies a third type of learning strategy that is concerned with the social and affective processes of learning. Examples 
include activities such as cooperation and redirecting negative thoughts.

O'Malley and Chamot (1990) used the theory from cognitive psychology to explain how the role of cognition can describe language competency. In this respect, they were moving away from the linguistic Universal Grammar paradigm of Chomsky and others, who maintain . language learning takes place differently than learning using regular cognitive skills (Spolsky, 1985; Skehan, 1991). Justification for using cognitive processes to explain the role of SLA and the use of strategies can be found in Spolsky's (1985) model on preference rules, which identifies three conditions that must be met to accomplish SLA: (1) necessary condition, target language input, motivation and practice opportunities, (2) gradient conditions, frequent interactions in the target language and opportunities to refine language skills, and (3) typicality conditions, traits that typically assist in the SLA, such as risk-taking and outgoing personalities. Further justification for using the cognitive processing model is based on: (1) the large amount of research in cognitive psychology which provides an established framework to look at SLA through, (2) the process orientation of the model which allows researchers to use it to investigate and explain strategies, and (3) the focus on language learning as a cognitive skill which gives researchers other ways to study how to improve language acquisition.

While learning strategies are not specifically addressed in cognitive psychology, they can be understood by looking through the information processing framework of learning. Short term memory (STM) is where 
new information is received. Because of its limited capacity, new information is either transferred to long term memory (LTM) or forgotten. Information is stored in LTM in one of two ways, as declarative knowledge or procedural knowledge (Anderson, 1982). According to Anderson's theory new information is acquired through a four-stage process: (1) selection (learners focus only on certain information and bring it into STM), (2) acquisition (information in STM is transferred to LTM), (3) construction (connections are made between information brought into STM and related information retrieved from LTM which increases understanding), and (4) integration (learners actively seek for information in LTM to bring it into STM). Since the first two processes delimit how much is learned and the last two decide what is learned and how it is organized, the importance of the role of learning strategies is that they can bring consciousness to the role of learning. Strategies can make explicit what otherwise might occur without learner awareness.

Anderson's theory distinguishes between two types of knowledge, declarative and procedural. Gagne, Yekovich and Yekovich (1993) define these terms as follows: declarative knowledge is what we know, it can usually be verbally expressed and is maintained in LTM in terms of meaning; procedural knowledge is our knowledge of how to do things and is represented in complex cognitive production systems, or 'IF-THEN' relationships. These two types of knowledge are learned in different ways. Declarative knowledge is stored in LTM in the form of schema and propositions, which can be retrieved by establishing linkages. Pieces of 
information, like grammar rules and vocabulary, can be learned quickly; but retrieval to STM, where they can be used, is cumbersome and slow because the limited capacity of STM would make it difficult for the learner to recall too many rules at one time. The function of procedural knowledge is to provide rapid performance of learned skills. For this reason it is important for language to become proceduralized. A computer analogy (Lachman, Lachman \& Butterfield, 1979) is that declarative knowledge is like stored data, whereas procedural knowledge is similar to the software program. Procedural knowledge occurs more slowly than declarative knowledge and can take place either with cued feedback or applying abstract principles from something already learned to new tasks (O'Malley \& Chamot, 1990).

To go from rule-bound declarative knowledge to more automatic procedural knowledge that reduces the burden on STM requires three stages:

1. Cognitive Stage: acquiring language skills begins where learners are introduced to new information and consciously interact with it, for example learning a new grammar rule

2. Associative Stage: two changes occur - errors in declarative knowledge are gradually eliminated and connections among components of various skills are strengthened

3. Autonomous State: performance becomes automatic and demand on STM or consciousness lessened (O'Malley \& Chamot, 1990). Learning strategies are linked to this theory because they involve 
specific cognitive processes which facilitate learning. Metacognitive strategies involve planning, selective attention, monitoring, and advance organization. Cognitive strategies include grouping, inferencing, elaboration, imagery, and deduction. Affective/social strategies influence which of the other strategies learners choose to focus on and integrate into new knowledge. These are the same activities procedural knowledge performs as it selects and develops declarative knowledge and transforms it into automated procedural knowledge. Learning strategies start as declarative knowledge, which then becomes proceduralized through practice. They do this by going through the cognitive and associative stages of learning, finally reaching the autonomous stage.

By showing how cognitive theory can be used to describe the influences of learning strategies on learning, O'Malley and Chamot have provided the foundation for applying this theory to research in the use of language strategies in SLA.

\section{Learning strategy definitions.}

The literature reveals that, in a broad sense, researchers agree on the definitions of different strategies. By looking at how different researchers define these categories, one can get a clearer picture of what they mean. Oxford and Nyikos (1989) state that, for information processing, the two categories learners use most are the cognitive and the metacognitive. According to Reid (1987), cognitive strategies are mostly automatic while metacognitive strategies provide learners with conscious management and 
control of their learning. Brown (1991) interprets cognitive styles as including how we think, receive information, and internalize it for later retrieval so that we can use it in the practical sense of communication. Cohen (1990) adds the idea of self-management. His definition also includes storing images in our memories that relate to what we already know so that we can easily retrieve this information. This definition of cognitive strategies extends beyond those in the Strategy Inventory for Language Learning (SILL) cognitive strategy group (Oxford, 1990).

Oxford separates this latter concept from her cognitive category and puts it into the category of memory strategies.

Some of the techniques Oxford and Crookall (1989) believe belong in the affective category are such strategies as reinforcement of successful learning and providing positive input to oneself regarding the ability to be successful - an 'I-am-capable-and-good-at-this' attitude will lead to more accomplishments than a 'I-simply-am-not-a-good-language-learner' attitude. Such positive attitudes can help L2 learners manage their emotions, while leading to improved attitudes and motivations. The use of good social strategies includes working with peers in an empathetic way and intermingling with native speakers. Finally, according to Ehrman and Oxford (1990), the six category groups can be divided into direct and indirect strategies which support each other and interact with each other. Direct strategies are used directly on the language and include cognitive, memory, and compensation strategies. The indirect strategies are supporting and managing methods which include metacognitive, affective, 
and social strategies. Oxford (1990) uses the analogy of the theater to explain the relationship. The actor, the direct strategies, works with the language for specific tasks and effects, while the director, the indirect strategies, works closely with the actor to assure that the performance has the best chance of being top quality.

\section{Classification of strategies.}

Because much of the of the research on LLSs has been done in the past decade and no agreement has been reached on a classification system, there are many ways of classifying strategies. Also, many data-gathering devices which identify strategies, such as group interviews, diary studies, and questionnaires, have been done on a case basis which makes research replication difficult (Skehan, 1991). Oxford (1992) points out that at least two dozen classifying systems exist, including ones which classify according to psychological function, particular language skills, and different types of learners. For example, Rubin's selections, as cited in Ehrman (1994), were based on tasks, goals, learning environment, and the personality and level of learning of the students she investigated. A 1989 study by Oxford and Nyikos using factor analysis showed five categories of LLSs: formal, rule-related processing strategies; functional practice strategies; resourceful, independent strategies; standard academic strategies; and conversational input elicitation strategies. O'Malley and Chamot (1990) place strategies into three major categories: metacognitive, cognitive, and social-affective. 
Bialystok's model includes four categories of learning strategies: inferencing, monitoring, formal practicing, and functional practicing. In her explanation, the strategy used by learners depended on three types of knowledge: explicit knowledge, implicit knowledge, and knowledge of the world (cited in O'Malley \& Chamot, 1990). Bialystok (1981) argues that implicit knowledge and world knowledge can be inferred. Monitoring, formal practice (such as classroom drills), and functional practice (using language in the world outside the classroom) can contribute to both explicit and implicit knowledge (Bialystok, 1981). She hypothesizes that strategies first used explicitly in a formal setting such as a classroom can later be converted to implicit knowledge and enhance students' ability to understand and use language spontaneously. This is in conflict with Krashen's (1982) Monitor Model, which states that we become secondlanguage proficient primarily through acquisition (implicit knowledge) not learning (explicit knowledge).

While Bialystok acknowledges the factors of motivation, aptitude, and personality, she believes that by consciously applying these cognitive/metacognitive learning strategies, learners gain mastery regardless of their personal characteristics and the learning situation. This view is supported by researchers (Chamot \& Kupper, 1989; Brown \& Palinscar, 1982) who believe that the problem of strategies is that they are learned for one task then fail to transfer to new tasks when both metacognitive and cognitive are not part of the language strategies skills students learn to use. Lack of the use of metacognitive strategies, in 
particular, can leave students without purposeful direction in their language learning endeavors.

In the last decade Rebecca Oxford's (1990) classification system has been used extensively. Her system incorporates most of the strategies mentioned by other researchers in a more detailed form. Furthermore, she has developed an information collecting instrument, the Strategy Inventory for Language Learning (SILL) which identifies six major categories of strategies: memory, cognitive, comprehension, metacognitive, affective, and social. This is a self-report survey L2 learners use to identify strategies they use in their own learning process. The development and standardization of this form has made it easier for researchers to gather data from students to use in their studies. A more thorough explanation of the SILL and Oxford's definitions of these categories are provided in the next section.

While acknowledging that Oxford's SILL survey has provided a good foundation for developing questionnaires, O'Malley and Chamot (1990) question the soundness of some of the strategies she has included.

In their view, her taxonomy includes strategies that go beyond those suggested in cognitive theory. They believe there should be a distinction between strategies that are used to learn language and those that are used to communicate. Other researchers (Tarone, 1981; Faerch \& Kasper, 1984) have distinguished between strategies used for learning, communication, and production. The motivation for using learning strategies is to gain linguistic competence. Production strategies are used 
to minimize efforts in certain communication goals, for example, using formulaic expressions and planning certain discourse strategies.

Communication strategies are the speaker's attempt to communicate without adequate resources in his/her interlanguage repertoire and include such techniques as paraphrasing, mime, circumlocution, avoidance, and appealing for assistance from the interlocutor (Tarone, 1981).

However, Skehan (1991) points out that there is considerable agreement between the schemes proposed by each of the above researchers because Oxford's cognitive and memory strategies are both part of O'Malley and Chamot's cognitive category. That leaves only the compensation category, which is characterized chiefly by communication strategies A justification for including this category in the SILL is that in Oxford's factor analysis research (Oxford \& Nyikos 1989) conversational input solicitation is one of the classifications which were identified. Also, since social skills strategies are part of O'Malley and Chamot's theory, one could argue that it would be difficult for an unskilled learner to apply some of the social strategies (such as cooperating with peers and proficient users or asking for help) without needing to use compensation skills, which Tarone (1981) classifies as communication skills (circumlocution, mime, etc.). Because one of the important goals of learning the target language generally includes being able to use it for communication purposes, this category, while not fitting into O'Malley and Chamot's, appears to be a reasonable part of a learning strategy taxonomy. 


\section{Strategy Measurements}

Information on what strategies L2 learners use can be obtained in several different ways. One method is to simply ask learners what kinds of strategies they use. This can be done through observations, diaries, questionnaires, interviews, or self-report surveys. The other method is to try to capture what strategies learners are using at the time they are doing tasks or soon after. These take the form of verbal reports.

In this study, a specific survey, the SILL (Oxford, 1990), was used to collect self-report data from all students involved in the study. Then, a think-aloud protocol was used to collect additional data on two of the subjects to assess which types of strategies were actually used on specific tasks and whether the subjects were using the strategies they had reported using on the SILL. The following provides some general background on these strategy measurements.

\section{Strategy Inventory for Language Leaming (SILL) Survey.}

The SILL is a structured survey where students are asked to report on how often they use 50 different strategies (Oxford, 1990). It has been used with language students in universities, schools, and government agencies in both foreign language and ESL classrooms around the world (Ehrman \& Oxford, 1989, 1990; Nyikos, 1990,1993; Oxford, 1990, 1990b, 1993; Oxford \& Crookall, 1989; Oxford \& Ehrman, 1993; Oxford, Lavine \& Crookall, 1989). Researchers have compared the results of surveys 
among different groups and conducted follow-up informal interviews to assure that students were not giving "socially desirable" answers (Oxford \& Nyikos, 1989). No biases have been found, thus substantiating the truthfulness of the answers. Oxford and Nyikos believe that this is because the students are guaranteed anonymity and the results are not used as any part of performance measures.

The SILL separates individual skills into six major categories: memory, cognitive, compensation, metacognitive, affective, and social. In general, Oxford's research (1989) has drawn the conclusion that students manage their learning through metacognitive strategies, control their emotions and attitudes through affective strategies, work with others through social strategies, put information into memory with memory strategies, use cognitive strategies for their analytical processing, and circumvent language limitations with compensation strategies. These are the six main groupings in the SILL. Listed within each of these groupings are specific strategies on which students can report their level of use. For example, under 'social strategies' students see a statement such as "I ask help from English speakers"; then, on a five-point Likert scale, going from 'never or almost never' to 'always or almost always', the students rate themselves.

The results of the SILL provide students with a profile of their individual strategy use. The student-profile categories correspond to the six major categories of skills but are stated in less academic terms: (a) remembering more effectively; (b) using your mental processes; (c) 
compensating for missing knowledge; (d) organizing and evaluating your learning; (e) managing your emotions; and, ( $f$ ) and learning with others. On the five-point scoring system, high use in the category group is reflected in a score of between $3.5-5$, medium use between $2.5-3.4$ and low use 2.4 or below (Oxford, 1990). Examples of the SILL and the learner's profile are in Appendix B.

While surveys are valuable tools for beginning to understand what learning strategies learners use, these inventories do not address how and when strategies are used but only that students are aware of using them. For more in-depth understanding of strategy use under specific conditions and with specific tasks, other methods are needed. One of those that promises to reveal more about actual strategy use is the think-aloud protocol, which is discussed next.

\section{Think-Aloud Protocol (TAP).}

The Longman Dictionary of Language Teaching and Applied Linguistics (Richards, Platt \& Platt, 1992) defines protocol as "a sample containing observation(s) of a phenomenon which is being described, observed, or measured" (p. 298). The TAP is a technique that helps researchers identify what strategies students are using as they complete activities. The students are asked to 'think-aloud', as if they were alone in the room, as they proceed through tasks and answer questions. In an attempt to understand the underlying cognitive processes of human thought, cognitive scientists conducted TAP analyses as early as 1920 , 
using note-taking to collect data from their observations (Ericsson \& Simon, 1993). With the invention of tape recorders during World War II, researchers were able to obtain and study verbalizations in their raw form. However, because of the popularity of behaviorism in the seventies, this form of research was not given much attention until the 1980's (Ericsson \& Simon, 1984).

This method has in recent years been introduced into second language acquisition research because human information processing is believed to be the same for this activity as in other thinking activities (Dechert, 1987). In a 1985 article, O'Malley and Chamot suggest that because there is no consensus on what constitutes a learning strategy, researchers need to be able to carefully identify which strategies are used on specific tasks. While researchers cannot observe what subjects are thinking, they can observe, record, and analyze what they say and do. The TAP provides the researcher with a tool to do this.

Ericsson and Simon's (1984) cognitive processing model looks at cognition as information processing. Central to their model is the idea that we can store a limited amount of information in short-term memory (STM) for a limited amount of time. From STM it can be transferred to long-term memory (LTM) which has a large capacity. Retrieval of information from STM can be accessed immediately, while that stored in LTM must be first transferred to STM before it can be used. The importance of this in obtaining reliable information about strategy use is that students' verbalizations from TAPs are from information that is immediate and being 
held in STM. Some of the assumptions of the Ericsson and Simon model are that:

1. The verbalizable cognitions can be described as states that correspond to the contents of STM.

2. The information vocalized is a verbal encoding of the information in STM.

3. the verbalization processes are initiated as a thought is heeded (i.e., noticed).

4. The verbalization is a direct encoding of the heeded thought and reflects its structure. (1993, pp. 221-222)

In other words, the subject doing the think-aloud encodes and verbalizes information in STM as he/she notices that it is there.

Before further discussion on the TAP, it is important to recognize that three types of learner report methods are used. In second language acquisition Cohen (1987) describes these as: (1) the self-report, which, like the SILL, simply has subjects describe what they do - these are described in statements like "I first review articles before I read them"; (2) self-observation, in which the subjects report their language behavior immediately after the event (introspectively) or somewhat later (retrospectively) - while the information is still in STM; and (3) selfrevelation, which takes place as the thinking process is occurring and the data is unanalyzed - the think-aloud report. Ericsson and Simon (1987) refer to these, respectively, as: (1) post-process observations, where information may be used from both STM and LTM; (2) performance observations, where no new information is added but subjects might label information that is in STM; and (3) process observations, where 
verbalizations may come from covert encodings and are spoken spontaneously and unedited.

One of the early concerns of researchers regarding the use of thinkaloud reports was that the process of thinking out loud might change the nature of the information gathered. It was important that the sequence of states of thought that are in STM remained unchanged when the thinkaloud procedure was used. Ericsson and Simon (1987) reviewed studies which compared the results of subjects doing TAPs to those who worked silently and found that there were no differences in the cognitive processes. The only observed difference was that those doing the TAPs took longer. Because of the longer length of the TAP process, thoughts have to remain heeded and subsequent thoughts cannot emerge until after the immediate verbalization has been completed.

Using TAP in second language acquisition brings in another dimension, that of the knowledge sources that learners make use of. If inferencing can be described as one of the main ways that people reach conclusions, then L2 learners must use linguistic cues, in combination with their other knowledge of the world. Haastrup (1987) points out that the cues they use can come from three main types: (1) interlingual - L1 loan words or knowledge of other foreign languages, (2) intralingual - based on the target language, and (3) contextual - based on the text and general world knowledge. An example of an interlingual clue might be if a student knows that the Spanish word "adios" means 'goodbye', when the word 'adieu' is introduced into a French lesson, the Spanish word provides a clue 
to this new word. Intralingual clues might include being able to extrapolate the meaning of an English noun such as 'obsession' when an ESL student already is familiar with the verb 'obsess'. World knowledge clues can be used in contextual analysis, where the student knows what the subject of the article is and can use this information to guess the meaning of unfamiliar words.

A major job the researcher must attend to before TAP data can be used is that of encoding transcripts. Ericsson and Simon (1993) stress that to reduce the risk of contaminating the output data, encoding decisions should be clear, specific, and based on theory and procedures held by the researchers. Encoders should specify the reasons for their choices clearly and these decisions should be made prior to conducting a TAP. Results of early studies of language learners were generally described with narratives, which made them difficult to replicate (Perl, 1985). Because of the needs of researchers to be able to see graphic evidence of the patterns and easily replicate studies, Perl developed an encoding system for her L1 writers that provided evidence of the frequency, underlying patterns, relative importance, and regularities of behaviors. Her system has been adapted and used by researchers in L2 (Raimes, 1985). Since then others (Abraham \& Vann, 1987; Oxford, 1990; Witte \& Cherry, 1994) have developed systems which can be adapted for use by researchers doing TAPs.

One of the interesting outcomes of Perl's use of her new encoding system was that she was able to take unsuccessful learners and show that 
their composing process showed consistency and regularity (1979). With careful use of her encoding system she discovered a similar behavioral system among these students that was consistent across writing sessions. When Raimes (1985) conducted a similar study on her L2 composition learners using Perl's code, she could evaluate the differences between unsuccessful L1 students and her successful L2 students - mainly that her students were not as preoccupied with editing. She speculated that, while L1 students were intimidated by errors, L2 learners expected to make mistakes and have the teacher correct them. As a result of the encoding system developed by Perl, Raimes was able to use Perl's system and to compare her subjects with Perl's. A possible drawback to the TAP that Cohen (1987) points out is that the verbalizations are limited to strategies which students are conscious of. Other problems that have been suggested are that subjects' verbalizations are inconsistent with their actions and that they are incomplete (Krings 1987). Krings refutes these problems by reminding us that, because of the concurrent nature of the TAP, as represented through Ericsson and Simon's cognitive processing model, verbalizations are made while the needed information is available in STM.

However, because of the difficulty in interpreting data even when the researcher has carefully developed an encoding system, TAP research is still used infrequently. The messiness of interpreting the data and the time intensive nature of the process have made it less popular than other types of research. Yet, as seen in the Perl and Raimes research, some valuable inferences can be made across studies when suitable encoding 
systems are available. In this study the encoding used to interpret the data was the strategy base identified in the SILL.

\section{Learner Variables}

How one uses LLSs and those which one chooses to use and not to use, are affected by many variables. Some of the more common ones include aptitude, motivation, anxiety, self-esteem, risk-taking, age, gender, culture, career choice, and personality type (Oxford, 1993; Oxford \& Ehrman, 1993). They also include what Oxford (1990b) refers to as learning style, i.e., general approaches students use that affect their responses to learning situations (Skehan, 1991). These responses can be voluntary or not and directly affect the strategies learners choose. Some of the dimensions of learning styles include analytical vs global processing, tolerance for ambiguity and sensory perception. The latter involves a learner's preference for auditory, visual, tactile, or kinesthetic learning experiences. The other two dimensions are discussed below.

\section{Global vs. analytical thinkers.}

A personality trait that shows a distinction among how students use strategies is whether they are analytical or global thinkers (Schmeck, 1988). Analytical students tend to favor cognitive strategies such as analyzing components of language and rule-learning, whereas global learners prefer strategies that allow them to look at the whole picture and use language in conversation (Oxford, Ehrman \& Lavine, 1991). 
One measure of whether a learner is a global or analytical thinker is field independence versus field dependence. Examples of this are pictures that are composed of dots that have a figure such as a face embedded in them. Field independents can easily pick out the figure, while field dependent have more trouble. Field independent learners tend to prefer more analytical, object-oriented information, while field dependents tend to prefer strategies that are more interaction-based.

\section{Tolerance for ambiguity.}

Tolerance for ambiguity refers to learners' ability to handle confusing situations (Ely, 1989). Hofstede (1994) states that one of the dimensions that can be used to classify cultures is the degree to which people avoid uncertainty. For example the French, Belgians, and Japanese demonstrate a high degree of uncertainty avoidance, while the English have a low degree. Those from cultures with a low tolerance for uncertainty prefer structured environments, while those with a higher tolerance for uncertainty choose to operate under less structured situations. Ehrman (1994) expands this definition by including the ability to hold contradictions in one's mind and integrate new information into existing schema. Ehrman relates risk-taking to this variable, with those learners that have a high tolerance for ambiguity showing more willingness to take risks - an essential part of progress. 


\section{Risk-taking.}

The personal variables of willingness to take risk and self-esteem are closely related to anxiety and tied to learning to the extent that students can effectively use affective learning strategies (Bacon \& Finnemann, 1990; Ehrman, 1994; Ehrman \& Oxford, 1995). Risk-taking is linked to ego boundaries (the extent to which individuals keep apart their mental, interpersonal and external experiences), which are a measure of selfesteem (Ehrman \& Oxford, 1995; Ehrman, 1994; Brown, 1987). Learners with thin psychological ego boundaries show more of a willingness to tolerate a certain amount of chaos in their language learning experience, while those with thick ego boundaries have a need for order and more structured learning environments. In Ely's studies (1988), risk-taking was positively related to relatively free use of language. Bacon and Finnemann's research (1990) also showed that the willingness to take-risk had a strong influence on how much learners engaged in target language interactions.

Several researchers (Ehrman, 1994; Sparks \& Granschow, 1991; Young, 1991, Horwitz, 1986) have looked at anxiety, feelings of tension and worry associated with arousal of our nervous system and its strong ties to language learning success. Brown (1987) found in some of his studies that not all anxiety has a negative influence on learning. In fact, he reported that some anxiety in certain situations (such as test-taking) correlated positively with success, particularly among high achievers.

Oxford has developed an Affective Survey (Ehrman \& Oxford 
1995) that measures motivation, beliefs about self, and anxiety. Using this survey, a significant correlation was revealed between anxiety and such performance measures as speaking up in class and proficiency. The negative kind of anxiety manifests itself particularly acutely in conversation. The anxious listener feels the need to understand every word spoken. It is speculated that the anxious speaker is dealing with issues of self- esteem that make it difficult for him/her to benefit from the conversational experience (Horwitz, 1986).

Young's research (1991) shows that, while different methods may be used to teach, learning is hindered unless students can learn to use strategies that help them minimize their anxiety and maximize their motivation. Students need to manage their emotional state in order to take in what they are hearing and to produce language. As a result of her studies, Young has identified three behavioral characteristics in students who are in a state of anxiety. She calls these: "1) arousal-mediated responses; 2) disaffiliative behavior; and 3 ) image-protecting behavior" (p. 429).

Krashen (1982) strongly supports the idea that, for learning to take place, students must learn to manage their anxiety. In his Affective Filter Hypothesis he proposes that learners' ability to take in information and progress in their L2 proficiency is in proportion to the anxiety they are experiencing; a strong affective filter prevents input from reaching the part of the brain responsible for acquiring language. Other studies (Oxford, 1993; Young, 1991) support Krashen's hypothesis and go on to say that 
since learning is directly affected by such things as motivation, anxiety, and attitude, it is important to try to identify and understand the strategies that manage these variables.

\section{Aptitude.}

In studying language students at the Foreign Service Institute (FSI) (Ehrman \& Oxford, 1995; Ehrman, 1994) aptitude, as measured by the Modern Language Aptitude Test (MLAT), showed a positive correlation with achievement, especially at the extremes. In Ehrman's study (1994) the weakest and strongest students scored lowest and highest on the MLAT. Aptitude was the strongest variable correlated with proficiency in these studies. Another interesting finding in these studies is they revealed a high correlation between aptitude and tolerance for ambiguity (a possible connection between a personality trait and aptitude).

In the opinion of the researchers this may be because the MLAT measures both fluid and crystallized abilities. Fluid abilities involve processing unfamiliar material and rapid adaptation of new information. Crystallized abilities have to do with working with learned skills. While weak students appeared to be overwhelmed by chaos, the stronger students seemed to find a way to assimilate new information and accommodate it into their existing schema (Ehrman, 1994). Memory strategies such as grouping and associating, the compensation strategies of guessing intelligently, cognitive strategies of analyzing and reasoning, and the metacognitive strategies of organizing and planning are all strategies 
that should help learners tolerate ambiguity and manage chaos.

\section{Motivation.}

In two studies (Oxford, Young, Ito \& Sumrall, 1993; Ehrman \& Oxford, 1995 ) motivation was the single best predictor of success in second language proficiency. In a third (Ehrman \& Oxford, 1995) affective and motivational strategies showed the second highest correlation with achievement (aptitude was first). One way that researchers have looked at motivation is on an integrative, instrumental continuum. When students want to learn language for practical reasons, such as career opportunities, they are said to have an instrumental orientation. Students who are learning a language because they have a strong interest in the native speakers and their cultures are said to have an integrative orientation. The general belief about this has been that those with an integrative orientation will be more successful language learners because their reason for wanting to learn the language goes beyond the mere acquisition of a new skill. They have the desire to use their new language skills to better understand the people and culture where the language is used.

Ehrman and Oxford's studies at the Foreign Service Institute showed a positive relationship between integrative orientation and achievement, but they did not find a negative correlation between instrumental orientation and achievement. They speculated that this relationship may have may have revealed itself because many of their students were Foreign Service Officers and their spouses, people who by their career 
choice had probably shown their interest in foreign cultures.

In a study (Lukmani, 1972) of Marathi-speaking high school girls in India the opposite was found. The highest achievers were those who had an instrumental orientation - a desire for better jobs. In another small study (Pearson, 1988) of Japanese businessmen living in Asia, anecdotal evidence showed that those who were interested in the culture and wanted to be a part of the community (integrative motivation), learned the language and considered their living abroad experiences successful while those who were not interested in mixing with the local population and kept to themselves didn't learn the language. These businessmen, incidentally, reported their experiences as stressful and unhappy.

Other factors that affect motivation include the students' need for achievement, fear of failure, likelihood of success, and the value the student places on that success, self-efficacy and the learning environment. No evidence in this review of the literature linked motivation to specific learning strategies; however, it would be expected from Oxford's explanation of her taxonomy of language learning skills (Oxford, 1990) that highly motivated students would, if they knew about them, use a high level of different strategies. Common sense would indicate that weakly motivated students might need to become more motivated before they would be interested in developing good strategy skills. Of course, in certain situations, for instance when there doesn't appear to be adequate improvement for the amount of work, the opposite could be true. If the weak students learned some good learning strategies and saw their effect, 
it could affect their motivation in a positive way. However, in the literature no positive correlations between motivation and specific strategies were found.

\section{Culture.}

According to a study by Reid (1987), culture and language weigh heavily into learners' sensory perception preferences. She found that, of those groups tested, Korean students were the most visual in their ways of learning, with Arabic and Chinese students also demonstrating a preference for this style. This could indicate that learning strategies such as using imagery and semantic mapping (see Appendix B) are preferred by students from these cultures. Japanese incorporated the least amount of auditory learning activities into their learning styles. Most ESL students strongly preferred kinesthetic learning as a major style. Strategies that favor physical action such as mime and gestures may assist these learns in SLA. Native speakers of English, among all groups, showed the least preference for tactile learning experiences.

In another study (Oxford, Young, et al., 1993) where satellite learning (a visual medium) was used, students beforehand had been evaluated regarding their perception preferences and motivation. Although those who preferred auditory input had been evaluated as being the most motivated, the visual preference turned out to be the most predictive of achievement. The researchers hypothesized that these results were a reflection of the lack of auditory and tactile learning activities for the 
students with these preferences.

From the studies it is evident that all strategies are not used universally and that culture plays a part in the selection of strategy use.

Gender, age, career choice, and bilingualism.

Gender, age, career choice, and bilingualism are four more variables which may affect learning and the kind of learning strategies learners prefer. In several studies (Nyikos, 1990; Ehrman \& Oxford, 1990; Oxford \& Nyikos, 1989) gender differences played a role in strategy choice, with women using more strategies than men. In these studies such concepts as color coding and pictures to enhance remembering (which may indicate a type of imagery strategy), and certain personality characteristics demonstrated some gender relatedness.

In the study by Nyikos (1990) a group of U. S. first year university students of German (135) were divided into four groups, each including male and female students. Each group was given the same list of nine German nouns, but different treatment conditions to help them memorize the nouns: (1) the association of color, (2) the association of pictures, (3) the association of colors and pictures, and (4) rote memorization. Significant differences were found in two of these groups. Women outscored men when color alone was used and men outscored women in the group that used both color and pictures. Also, men who had treatment three (colors and pictures) outscored the men in groups one and two who had the treatments of color only or pictures only. 
In other studies (Oxford, Young, et al., 1993; Oxford Nyikos, 1989; Ehrman \& Oxford, 1989) females showed a nearly significant correlation with the strategy categories of cognitive, social, and affective.

Ehrman has had a unique opportunity to study the effects of age on learning and strategy choice because she is with the Foreign Service Institute (FSI), a language training center for the State Department and other government agencies and her subjects have included a range of ages. In some of her research, much of it with Oxford (Ehrman \& Oxford, 1995; Ehrman, 1994; Oxford \& Ehrman, 1993; Ehrman \& Oxford, 1990), she has found age to have a limited negative correlation with language learning success, but no strategy preferences were identified on the variable age.

Career choice has appeared in studies as a variable that shows some correlation with strategy choice. At the FSI, professional language trainers demonstrated a wider use of strategies and a preference for global thinking (big picture strategies and conversation) than teachers and students. Ely (1988) found that, while science students prefer more traditional classroom learning, engineering and business students prefer less structured learning opportunities.

Finally, there is some evidence (Nayak, Hansen, Krueger \& McLaughlin, 1990) that bilingual adult students had superior overall language abilities and were able to adjust their learning strategy use to task requirements better than monolingual adult students. They show more flexibility in such strategies as rule-discovery, memory, and restructuring their existing knowledge to apply to new information. 


\section{Personality type.}

Another learner variable that affects strategy choice is personality type. Personality type is one of the important factors that determines a student's learning style. Ehrman and Oxford (1990) define the term 'learning style' to encompass the habits and preferred patterns of dealing with incoming new information that people use when engaged in learning activities. They posit that styles may be at the root of an individual's natural strategy preferences in the Meyer Briggs Type Indicator (MBTI). Oxford (1990b) calls learning styles the 'missing link' to understanding the language learning process because they are so individualistic.

Research carried out by Ehrman and Oxford (Ehrman \& Oxford, 1995, 1990, 1987; Ehrman, 1994; Oxford \& Ehrman, 1993; Oxford, 1990b) on the relationship between personality type and strategy choice has shown some meaningful correlations between these two variables. One instrument used for measuring personality types, MBTI, is based on the theories of Carl Jung, which suggest "...that individuals have preferences that affect what they pay attention to in a given situation and how they draw conclusions or make decisions about what they perceive" (Ehrman \& Oxford, 1989, pp. 2-3). Within the MBTI there are 32 possible personality types. In the classroom (and outside) each type approaches learning in a different way. The reason for Oxford and Ehrman's interest in personality types is that, if learners know their type and teachers recognize the multiplicity of their classroom students' language learning preferences, they can use this knowledge to help make classroom 
experiences more effective.

Ehrman and Oxford define styles as being measured on four continua that constitute the four primary personality determinants in the MBTI. The first is the extrovert/introvert continuum, which indicates how we take in energy; the extrovert is energized by being among others, the introvert by being alone. The second pair is sensing/intuition and deals with how individuals gather data; sensors are practical, factual and more linear, while intuitors are more global. Thinking-feeling is the next continuum; it has to do with how we process the data we have gathered. Thinkers are more impersonal and objective while feelers take into account the situation and those involved. Finally, there is the judging/perceiving continuum. This deals with how we prefer to order our lives and it can be understood by looking back at the second and third continuum pair. Perceivers are open ended and like to keep taking in information--the function of continuum two. Judgers look for solutions, answers, and closure--the function of continuum three.

The results of the study by Oxford and Ehrman (1989) do indicate some interesting correlations between personality types and strategies. Extroverts use more affective strategies and visual strategies. Intuitors use more searching and communicating for meaning strategies and make use of formal model building in their learning. Feelers and Judgers use more general study strategies. In addition, there are two gender related pieces of data: females use a greater number of strategies, and the majority of feelers are women while the majority of sensors are men (Ehrman \& 
Oxford, 1989). The first of these gender differences is also confirmed in other studies that were discussed in the previous section.

\section{Successful/Unsuccessful Leamers}

When researchers looked at the difference between weak and strong language learners with similar MBTI profiles, they found that successful learners, even when having a preference for one learning style, were able to adapt to learning tasks outside their chosen style while the unsuccessful learners were less able to (Ehrman \& Oxford, 1990). Rubin, as early as 1975 , identified the characteristics of the successful learner, as discussed earlier in this chapter. In a study by Chamot and Kupper (1989) the less effective students were analyzed as having used fewer strategies and using them inappropriately for the various tasks. Vann and Abraham $(1989,1990)$ in their research involving unsuccessful learners found that, while their subjects used as many strategies as the successful learners, the problem seemed to stem from the fact that they did not adjust their strategy use appropriately for different tasks. This is one of the reasons given by strategy researchers for concentrating on the cognitive and metacognitive learning strategies such as analyzing and recognizing formulas (cognitive) and planning and monitoring (metacognitive) (Oxford, 1990).

Other factors that have been shown to affect learners' success are that less skilled learners use fewer strategies, pay less attention to which ones they use together, and are less creative in choosing strategies (Oxford, 1993). In addition, Reiss (1981) found that less successful 
language students do not seem to be aware of the strategies they use and/or have not consciously developed any approaches to L2 learning that might help them in a variety of learning activities. This very fogginess around what it is they are attempting to do and how to do it may contribute to the less successful language learners' disappointing outcomes. Nyikos and Oxford (1993) also report that unsuccessful learners demonstrate a lack of strategy-related awareness.

The successful learner, on the other hand, seems to use more strategies and does a better job of combining them (Oxford, 1993; Chamot \& Kupper, 1989). The Chamot and Kupper study revealed that successful learners were more purposeful in their approach to both task and strategy use, and they monitored for overall comprehension and production rather than for smaller components. While some strategies were used with all tasks in their study (monitoring and elaboration), others were used for specific tasks. For example, more inferencing was used for reading, while planning, composing, reviewing and summarizing were used with writing tasks. On listening tasks the effective students studied the native language composition questions ahead of time and used inferencing to help them predict what they were going to hear. They also used selective attention strategies rather than trying to understand everything they heard.

Other research with beginning and intermediate students (O'Malley $\&$ Chamot, 1985) revealed that the intermediate students were able to use more metacognitive strategies. They attribute this to the possibility that, as students gain more proficiency, they are able to concentrate on other 
aspects of their learning efforts. In this study, the lower level students used simpler strategies (repetition and note-taking) while the intermediate students approached more difficult tasks using more complex strategies. They found that metalinguistic awareness, the ability to think and talk about language and to focus on the forms, was helpful to these learners. It allowed them to make comparisons between L1 and L2 and to self-monitor and self-correct.

Bialystok (1981) worked with some learners who had been introduced to language strategy use. She found that simply quantifying how many strategies learners use will not allow us to predict achievement. The important element was the specialized effect that strategies have. This specialization has two consequences: (1) time spent using certain strategies is more effective than time spent using others, (2) the language activity determines what strategy will be most helpful. In her study the strategy most likely to lead to high achievement was functional practice (communication). Additional formal practice (doing one's lesson) no longer facilitated learning after a certain point. A 'ceiling effect' was found with students who continued to use this inappropriate strategy in greater amounts as they tried to improve their skills.

In her work, Oxford (1990b) has come to the conclusion that successful learners use a variety of learning strategies that work with the material and the learning task they are involved in. These are adapted to fit the task and to fit the students' own goals and needs. They are also selected with an appropriateness to the level of learning. For instance, 
good learners might rely on one strategy, such as learning vocabulary with the use of flash cards at an early stage in their L2 experience, and abandon this strategy as they get farther along.

O'Malley and Chamot (1990) believe that it is important for researchers to be able to identify the type of learning strategies that are most effective in helping learners succeed in various tasks. Since learning strategies have proved effective in first and second language reading comprehension, they are a potentially powerful tool in helping students who now use strategies inefficiently. They stress the importance of including lower ability students in future research studies to assure that good strategy use can be available to students at all levels.

\section{Summary}

After looking at learning strategies, a wide variety of learner variables, and the differences between successful and unsuccessful learners, several ideas seem clear: first, there are many variables that may affect how learners choose the strategies that they use; second, there are different learning styles that lead students to prefer certain strategies over others; and, finally, there is some evidence that those strategy choices are different for successful and unsuccessful learners.

From this it seems reasonable to predict that, if learners were more aware of the variables that affect their strategy choice and if they were presented with evidence of the different kinds of choices that are made by good and bad learners, they might be interested in looking more critically 
at the strategy choices they make. This, of course, is the ultimate hope of the researchers in these studies. As more evidence becomes available from research, teachers and students will be able to use it to help students learn to make strategy choices that will enhance their chances of gaining L2 proficiency. 


\section{CHAPTER III}

\section{METHOD}

Seventeen English as a second language (ESL) students participated in the first part of this study by completing a survey which identified the types of learning strategies they use as they engage in activities that are related to their attempts to gain proficiency in English. Two students from this group were selected to participate in the second part of the study. These two students were asked to complete a series of activities and to think-aloud as they worked. The rationale for the first part of the study was to see if there was a correlation between the students' strategy choice and their proficiency in English. In the second part of the study the researcher investigated whether the more proficient student used different strategies for completing tasks than did the less proficient student.

The survey used was the version of the Strategy Inventory for Language learning (SILL) designed for speakers of other languages learning English (Oxford, 1990) in that the language in the survey is simpler and easier for non-native speakers to understand. One of the important issues that the SILL does not address is the cultural preference for certain learning strategies. Because of this some of the conclusions drawn from the SILL survey data in this study may not accurately reflect the most useful strategies for learners whose cultures are significantly different than students in the U.S. However, all of the strategy taxonomies that were available for the study have been developed in the 
U.S. and, thus, pose similar problems . Since the SILL has been tested and found both reliable and valid in the studies thus far done, it is the one chosen for this study.

The think-aloud sessions were designed to follow the protocol procedures suggested by Ericsson and Simon (1993). Activities used to collect verbal data in the think-aloud protocol were similar to those used by earlier researchers (Abraham \& Vann, 1987). The activities were identical for both subjects.

The subjects were international or immigrant students studying English as a second language in an ESL program at a university in the northwest. They were all class members of the top two levels in the university's ESL program. The goal of most of the students in the program was to become proficient enough in English to be able to enter into one of the degree programs at the university.

An audiotaped pilot study on the think-aloud protocol was conducted, using one of the students from the advanced level . Pilot study observations revealed that the protocol was longer than was first thought necessary. Based on this discovery, several changes were made: the number of tasks was reduced from four to three; one of the sections, the verb tense activity, was shortened; and the initial interview questions and practice activities were not recorded during the actual think-aloud sessions. Also, the quality of the audiotaped session was poor. As a result of this, transcribing the data from the pilot study had to be abandoned. To correct this problem, the actual sessions were done on 
equipment in the university's learning laboratory in one of the sound rooms. To assure that the subject in the pilot study understood the process, several practice tasks were given prior to the actual exercises. This gave the subject a better opportunity to understand what was expected and to get him used to thinking aloud. Prior to the practice tasks instructions were given, describing the think-aloud protocol and specifically stating what was supposed to be done while completing the activity. These instructions were written out and read to the subject. He was then asked if he had any questions. One practice task was eliminated, a double-digit multiplication problem, $24 \times 36$, when it proved too difficult for the subject to do in his head. The rest of the practice tasks and the written instructions were unmodified in the two actual sessions. Because of these changes and the difficulties surrounding transcribing the data collected in the pilot, the results were not included in this study.

\section{Subjects and Sampling Procedures}

The subjects for the first part of the study were seventeen ESL students enrolled in writing classes at the two highest levels of the program, upper intermediate and advanced. The subjects in the second part of the study were both from the upper intermediate class. When students enter the ESL program at this university they are assigned to one of four levels on the basis of a series of standardized tests: The retired form of the Michigan Test of English Language Proficiency, the listening portion of the Comprehensive English Language Test (CELT) and the Test 
of Written English (TWE). All of these are the non-secure versions of the tests given by qualified staff in the ESL program. The Michigan and the CELT are multiple choice tests with objective answers. The TWE is a spontaneous timed writing sample that is graded holistically by the coordinator of the ESL program. The scores on these tests, along with course grades and teacher assessment, are the evaluation tools used to determine if a student is sufficiently prepared for the next level.

Of the students in the study, the first language of five was Arabic and eight of the students were from East Asia. The first languages of the remaining four were Spanish, Fon (from Niger), Italian, and Russian.

Teachers in the program were consulted regarding the reading capabilities of the students in the different levels of the ESL classes since it was important that the students be capable of interpreting and answering the questions on the SILL survey. It was decided that the students in the two highest levels would be most suitable. To enter the upper intermediate level, a student must have scored between 65 and 74 on the Michigan and CELT tests and have obtained a score of three on the TWE.

To enter the advanced level, a student must have scored between 75 and 85 on the Michigan and CELT tests and four on the TWE. Since the research was composed of two parts, the procedures for each will be discussed separately.

\section{SILL Survey Study Procedures}

The SILL is a self-report survey that asks students to assess the 
frequency of use for the strategies listed. There are fifty strategies listed on the survey and they are divided into six strategy categories: memory, cognitive, comprehension, metacognitive, affective and social. These categories are not named on the survey, but merely divided into parts $\mathrm{A}$ through F. Students are given a statement about strategy use then asked to report their use of that strategy on a Likert-scale measure, with one of five options. Their answers are to be put on a work sheet provided at the time they are given the survey. The following is a copy of the example given on the top of the survey to show the students how to complete it

\section{Example}

1. Never or almost never true of me

2. Usually not true of me

3. Somewhat true of me

4. Usually true of me

5. Always or almost always true of me.

Read the item, and choose a response (1 through 5), and write in the space after the item.

I actively seek out opportunities to talk with native speakers of English

You have just completed the example item. Answer the rest of the items on the work sheet. (Oxford, 1990, p. 294)

The SILL is a structured survey that can be objectively scored and analyzed. It has been field-tested with students around the world with learners of many languages and has been shown to be valid and reliable 
(Oxford 1990). A score between 5 and 3.5 is considered to demonstrate a high level of learning strategy use. A score between 3.4 and 2.5 shows medium use of learning strategies. Low strategy use is 2.4 or under. A copy of the SILL is found in Appendix A and definitions of all the strategies in the six categories are found in Appendix B.

The survey, work sheets and student profile sheets were left with the teachers with written instructions. The teachers talked with the students during their regular writing classes about the SILL. The students were told that there were no right or wrong answers to the survey, that their survey would be kept confidential and would in no way influence their grades. The survey and work sheet were handed out to the students, the instructions were read aloud to them by the teacher, and questions were answered. The students completed the survey at home with answers being marked on the work sheet. After they answered all the questions the students were instructed to calculate their score by following the simple mathematical directions given on the work sheet. The next day during class the surveys were collected. Then, the students were given the profile-of-the-results form. This form is the student's copy and is also divided into six groups. However, the groups are described in language that is less academic and meant to be easier for the student to understand. For instance, the memory group is called "remembering more effectively," and the cognitive group is called " using all your mental processes." In class they transferred the work sheet answers to this form and the work sheets were then collected from the students. The profile-of-results forms 
were kept by the students. When this activity was completed, the students and teachers discussed the profile and questions were answered. The completed surveys and work sheets were collected from the teacher by the researcher. Several days later the researcher talked to the students, answering questions about the study and learning strategies. Those agreeing to participate in the survey were asked to sign an informed consent agreement. Only those who signed the consent form were included in the study. All the work sheets were checked for mathematical accuracy.

To measure proficiency, the retired non-secure version of the Michigan Test of English Language Proficiency and the listening portion of the Comprehensive English Language Test (CELT) were used. These tests were both given by the instructors in the classes at the end of spring term 1995 as part of the ordinary evaluation procedures of the ESL program. Only those students who had taken the SILL and these two tests were included in the study. The composite student profile from the SILL and the results of the students' scores on these two tests were statistically compared to answer the first three research questions.

\section{Think-Aloud Protocol (TAP) Procedures}

Selection of the subjects.

Subjects for the TAP were selected from among those students who were willing to let the researcher use their SILL survey and the results of the proficiency measures, the Michigan Test of Language Proficiency and 
the listening portion of the CELT. The researcher asked for volunteers from both the upper intermediate and advanced writing classes but most of the volunteers were in the upper intermediate writing class. Therefore, it was decided to use two female students from the upper intermediate writing class. The first language of one of the subjects was Russian, the other's was Japanese.

From the answers to questions asked during the interview section of the training period it was revealed that both had been studying English in the university's ESL program for nine months. The Russian subject had studied English two years before coming to the U.S. and it was her only other foreign language. While the Japanese student had studied English for six years, she stated that she did not apply herself to learning it and had almost no ability to use the language until she came to the U.S. Besides English she is fluent in Mandarin. Both students stated that when they arrived in the U.S. they were incapable of having a conversation in English. By the time of the think-aloud study they were both fluent and capable of long sophisticated conversations in English.

The objective of this part of the study was to see if the strategies used by a proficient learner and a less proficient learner were different as they completed different tasks. To that end, the two subjects were selected from the a list of volunteers in the upper intermediate level who had reported similar strategy use on the SILL survey, but were performing at different proficiency levels. The overall average on the SILL survey was exactly the same for the two subjects, 3.5 , a score in the high range. 
While there were some differences on the scores of the separate sections of the survey, the general profile of the two subjects was similar. In other words, they both reported similar strategy use on each of the categories of the SILL: if one reported high in a category, so did the other; if one reported a lower use in a category group, the other one did too. Their performance on the proficiency exams indicated that one of the students was more proficient than the other. The more proficient student had scored 70 on the Michigan test and 92 on the CELT. The less proficient student had scored 60 on the Michigan test and 80 on the CELT. Before final selection was made, the researcher conferred with their writing class teacher, who agreed that these two fit the profile of learners which the researcher was seeking.

This part of the study was similar to one done by Vann and Abraham (1987). The composition exercise described below was the one they used. They also used a verb tense cloze exercise, but not the same one used in this study. The one main difference was that this study used one successful and one unsuccessful learner with similar SILL profiles, while their study used two unsuccessful learners. By using a Think Aloud Protocol, Abraham and Vann (1987) were able to identify the incompatibilities between those strategies that their learners used on specific tasks and the kinds of strategies that would have been better to use. However, their study did not use the SILL. The value of combining the results of the SILL and the Think Aloud data was that the researcher was able to compare the subjects' reported strategy use with those 
strategies which were actually used by these two learners on specific tasks.

\section{Data collection procedures.}

The tasks to be used for the TAP were selected with the cooperation of the coordinator of the ESL program at the university. The objective was to choose tasks that were of sufficient difficulty but not beyond the capabilities of the two subjects. Three tasks were selected for the protocol.

The first task that the subjects were asked to complete was a verb tense exercise that included a variety of verb tenses. The verb to be used was supplied and the subjects were asked to provide the appropriate tense within the context of the sentences. The second task consisted of a list of fifteen words that the subjects were asked to memorize in five minutes. For this exercise, they were given note pads to write on during the fiveminute memorization period. After the five minutes, the students' notes and the list were taken away and they were asked to reproduce on a sheet of paper as much of the list as they could remember. The last exercise was the most difficult of the three. They were given a composition task. They were to play the part of a traffic officer who had been at the scene of an automobile accident the previous day and taken notes on what she had seen after the accident. They were given a drawing of what took place during the accident. Their task was to coordinate this information and make a report on how the accident occurred. Copies of the three tasks are found in Appendix C. 
Prior to the TAP sessions, the researcher talked with each of the subjects separately to explain what they would be asked to do. The meetings were informal and the students asked questions about the types of activities they would be doing and what the researcher expected from them. At the end of these meetings, times that were suitable for the subjects were agreed upon. The subjects were told that the whole process could take from three to five hours. Actual time for the interview, combined training and set of tasks took about one and one half hours. During the first hour the TAP would be explained in more detail and some practice tasks would be done so that they could get used to thinking aloud. After that there would be a break, then the actual tasks would begin. It was agreed that the best time was on Friday afternoon, since there were no ESL classes during that period. The TAP sessions were scheduled on consecutive Fridays in one of the sound rooms in the learning lab at the university.

Both TAP sessions were conducted in the same way, as nearly as possible. The sessions took place in a small sound room. The subjects sat at a desk with a microphone in front of them. After the interview the researcher sat in a chair that was located to the side and behind the subjects. The entire session was audiotaped, although only the data from the actual sessions were transcribed and used in the analysis. The reasons for audiotaping the interview and practice activities was to allow the subjects to get used to being taped and to allow the researcher to check the quality of the audiotape material. The audiotaped data from the interviews 
and practice sessions were not saved.

First, the researcher interviewed the subjects. This interview served the dual purpose of collecting general background on the subjects and helping them relax. The interview was short, approximately five minutes, and the questions were the same for each subject. Effort was made by the researcher throughout each session to create an environment that was friendly and stress-free. It was emphasized from the beginning that no one would see their answers to the activities and, in fact, their answers were not the main focus of the session. The real objective of the researcher's study was to see what kinds of strategies they used as they completed the three tasks. This was the reason it was important that they talked out loud during the whole session. The second step of the process was the reading of the instructions, which explained what 'thinking aloud' meant. The researcher told them that she would be sitting behind them but if they had a question about a vocabulary word they could ask her the meaning. They were also informed that if they stopped talking for even a few seconds, the researcher would remind them by saying, "think aloud." During the session, every effort on the part of the researcher was made to say these "think aloud" reminders gently and encouragingly.

After the interviews, the reading of the instructions and the answering of questions, the practice session began. They were advised that in the practice tasks and the tasks for the actual data collection they could take as long as they needed to complete the tasks. There were three practice tasks. First the subjects were given a multiplication problem to do 
in their head out loud, $18 \times 7$. Next they were given an anagram and asked to find the common English word that the letters represented. An example of this was provided to assure they understood what an anagram was, KOBO (book). Then they were given the letters NPEPHA (happen) to identify as they thought aloud. After these two tasks they better understood how thinking-aloud worked. The final practice task was a longer cloze activity where they were asked to select the appropriate article to use in a sentence. Upon completion of all the practice tasks, they were asked if they wanted a long or a short break before the actual sessions began. Both chose a short break. During this time we chatted casually and relaxed. Both subjects displayed friendly, social personalities and were capable of sophisticated conversations in English. It was during these breaks that the researcher discovered that when the subjects had arrived in this country only nine months earlier their interaction capabilities in English were very low.

After the break the three tasks were given, without a break in between. These three tasks have been described above. While the researcher primarily used the term 'think aloud' when there were periods of silence, she did vary her prompting occasionally with terms like 'say what you are thinking' and 'what are you thinking?' Most of the time, however, the 'think-aloud' or 'speak aloud' prompt was used. The subjects were not rushed and at the end of each task were asked if they were through. When the subjects asked how they had done, the researcher advised them that they had 'done fine' or 'you are doing just great' or some similar comment. 
Only once, during the practice session, did she comment on the correctness of an article use when asked after that task had been completed. During the last task, when both had extended periods of difficulty trying to understand what was expected of them, the researcher indicated for them to use both the picture and the policeman's notations to try to report how the accident had occurred. This was the only assistance the subjects were given on any of the three tasks. The same process was used as in the practice session. While the subjects had to be reminded quite frequently to 'think aloud', most of the times that they were reminded, they did start to verbalize their thoughts immediately. While they thought the process of doing the tasks and thinking aloud the whole time was difficult, neither one appeared nervous and both cooperated fully.

\section{Data interpretation.}

The next step was to transcribe the data. This was done by a typist hired by the researcher. The transcriptions were then checked carefully, using the audiotapes, by the researcher to ensure their accuracy. To establish inter-rater reliability for the analysis of the TAP, first an experienced language teacher and I analyzed one task for the data, checking with each other and discussing our decisions as we proceeded. For example, on the memorization exercise, was there inter-rater agreement on when a grouping strategy or a associating/elaborating strategy (the memory strategy group) was being used. Did both raters agree when a strategy such as repeating (cognitive group)? Definitions of 
all the strategies in the six groups can be found in Appendix B. On the next task, we analyzed the section separately, doing about one to two minutes at a time, marking our raters' sheets at the end of each period. After the task was rated, we tallied our separate ratings and then discussed our differences. Inter-rater agreement was calculated at $91.8 \%$.

The remainder of the tasks were analyzed alone by me, the researcher. Strategies were noted by simply making a mark next to the strategy on a work sheet that listed all the strategies. The strategies definitions in Appendix B were often referred to as I was making decisions. In the beginning, I attempted to use one-minute sections to analyze. However, this immediately proved to be too long a period. The strategy that was adopted was to listen until one or two strategies were noted, stop the recorder, and decide which strategy was used. I often rewound the tape to listen again and make sure that I agreed with my own rating. At the end, when I discovered large differences in several strategy groups, I selected one task and reviewed both subjects to assure that my ratings on both students had been accurate. The analysis of the reviewed section against the original ratings showed that they were the same, within one strategy. Samples from the transcripts of the TAP with the strategies noted on them are in Appendix D.

\section{Statistical analysis.}

Statistics from the SILL and proficiency tests were calculated by a statistician in the statistics department of the university. Because of the 
small number in the sample, a Mann-Whitney U non-parametric measure was used to determine if there were significant differences between mean scores of proficient and less proficient users. A proficient learner was defined as one having a score of 70 or more on the combination of the Michigan and CELT tests. A less proficient learner received less than 70. The tests were weighted, with the Michigan scores given a two-thirds value and the CELT score a one-third value. These data were used to answer research questions one, two, and three.

The second part of the survey was not conducive to inferential statistical analysis because there were only two subjects involved. Therefore, percentages and tendencies were analyzed by the researcher to provide descriptions of the differences between the two subjects and to answer research questions four and five. With only two subjects were used no inferences could be made regarding validity. Also, because of the element of personal interpretation of the strategies used by the subjects, it would be difficult to draw any conclusions regarding the reliability of the data. 


\section{CHAPTER IV}

\section{RESULTS}

In this chapter the results of the data collected in the two parts of the study are reported. First the results of the SILL survey will be analyzed and discussed; then, the results of the Think-Aloud Protocol will be examined.

\section{Research Question One}

The first research question asked whether English as a second language (ESL) learners who are proficient will have a strategy-use profile that shows their strategy-use level is at a score of three or more in all major strategy categories: memory, cognitive, comprehension, metacognitive, affective, and social (source: Oxford's Strategy Inventory for Language Learning (SILL) survey, Oxford, 1990).

To determine whether the differences were significant, the MannWhitney $U$ and Wilcoxon Rank Sum W test was used to compare the means of the two groups, proficient and less proficient learners, on each of the six strategy categories. This test was chosen because it can be used with small samples where the groups are not of the same size, and the data can be nonparametric and are not required to have normal distributions. The total number of samples in this study was seventeen, twelve in the PL group and five in the LPL group. However, the test does assume "...that 
the scores in each of the two samples are independent and that the distribution of the two groups are similar except for central tendency" (Brown, p. 175, 1988). The test compares the two groups on the basis of their rank above and below the median to see if that difference is significant. It uses a two-tailed probability, to indicate that the differences can be non-directional. This works with ordinal data and was considered appropriate, since the answers on the SILL survey (from 'almost always' to 'never') can not be considered interval data. The alpha decision level of .05 was selected. Table one shows the relevant results of the test.

\section{TABLE I}

SUMMARY OF STATISTICS FROM SILL

\begin{tabular}{|c|c|c|c|}
\hline Strategy & Mean PL & Mean LPL & 2-tailed I \\
\hline Memory & 3.2167 & 2.6400 & $.0221^{*}$ \\
\hline Cognitive & 3.5667 & 3.4800 & .4582 \\
\hline Compensation & 3.5083 & 3.7000 & .3115 \\
\hline Metacognitive & 4.0083 & 3.8000 & .3962 \\
\hline Affective & 3.1333 & 3.1800 & .7094 \\
\hline Social & 3.8000 & 3.7400 & .8738 \\
\hline SILL Avg. & 3.5667 & 3.4400 & .4900 \\
\hline
\end{tabular}

* = Statistically Significant at $\mathrm{P}=<.05$ Maximum score on SILL $=5$

PL = Proficient Learners $(n=12)$ LPL $=$ Less proficient learners $(n=5)$

The full results of the statistical analysis are in Appendix E. The large 
difference between the two sample sizes and the small total number of samples does raise issues of validity that need to be considered. The results might not be meaningful for because of this.

The only strategy that showed a significant difference between the two groups was memory. With a mean for the proficient group at 3.2167 and the mean for the less proficient group at 2.6400, this strategy did reveal a statistically significant difference $(\mathrm{P}=.0221)$. From this, it can be concluded that, for the category of memory strategy, the mean of proficient learners (PLs) is greater than the mean of less proficient learners (LPLs) and PLs use significantly more memory strategies than those in the LPL group. None of the other strategy categories showed significant differences between the means of the two groups.

Although there was no statistically significant difference between the means of the proficient and LPLs in the other five categories, PLs uses slightly more strategies in the cognitive $(\mathrm{P}=.4582)$ and metacogntive $(\mathrm{P}=$ .3962) strategy groups. However, the reverse was true for compensation, where the LPLs had a higher mean than PLs at roughly the same tendency $(\mathrm{P}=.3115)$ as in the cognitive and metacognitive categories. Also, LPLs had a slightly higher mean in the affective category. But, at a $\mathrm{P}$ value of only .7094, it clearly indicated that there were almost no differences between the means of the two groups for that category. Similarly, in the final category, social strategies, PLs had a mean that was slightly higher than LPLs, but with a P value of only .8738 , little difference was revealed between the two groups. 
The average for the two groups on the entire SILL shows that the more proficient learners, with a mean of 3.5667 , do use more strategies than LPLs, who had a mean of 3.4400. However, with a $P$ value of only .4900 , this difference was not statistically significant. Therefore, except for the memory category, the answer to research question one is that these two groups did not differ in their reported strategy use. However, it is important to remember when looking at the results of the analysis that if a bigger sample size had been used there is the possibility other significant differences would have been revealed.

\section{Research Question Two}

This research question asked whether proficient learners will use more metacognitive and affective strategies than less proficient learners. While mean scores in all the categories were higher than three for the proficient learner group, it should be noted that the raw data on the individuals in this group did show some scattered scores of less than three in some of the categories. The raw data are reported in Appendix E. Furthermore, the LPL group also had mean scores of over three in all categories except memory, the one strategy that revealed a statistically significant difference between the two groups. Both groups had an overall SILL average of more than three. The statistical analysis did not show that PLs use more metacognitive and affective strategies than LPLs. 


\section{Research Question Three}

This research question asked if the difference in the number of metacognitive and affective strategies, as measured by the SILL, used by proficient learners and less proficient learners would be greater than the difference in the number of strategies they use from the other categories.

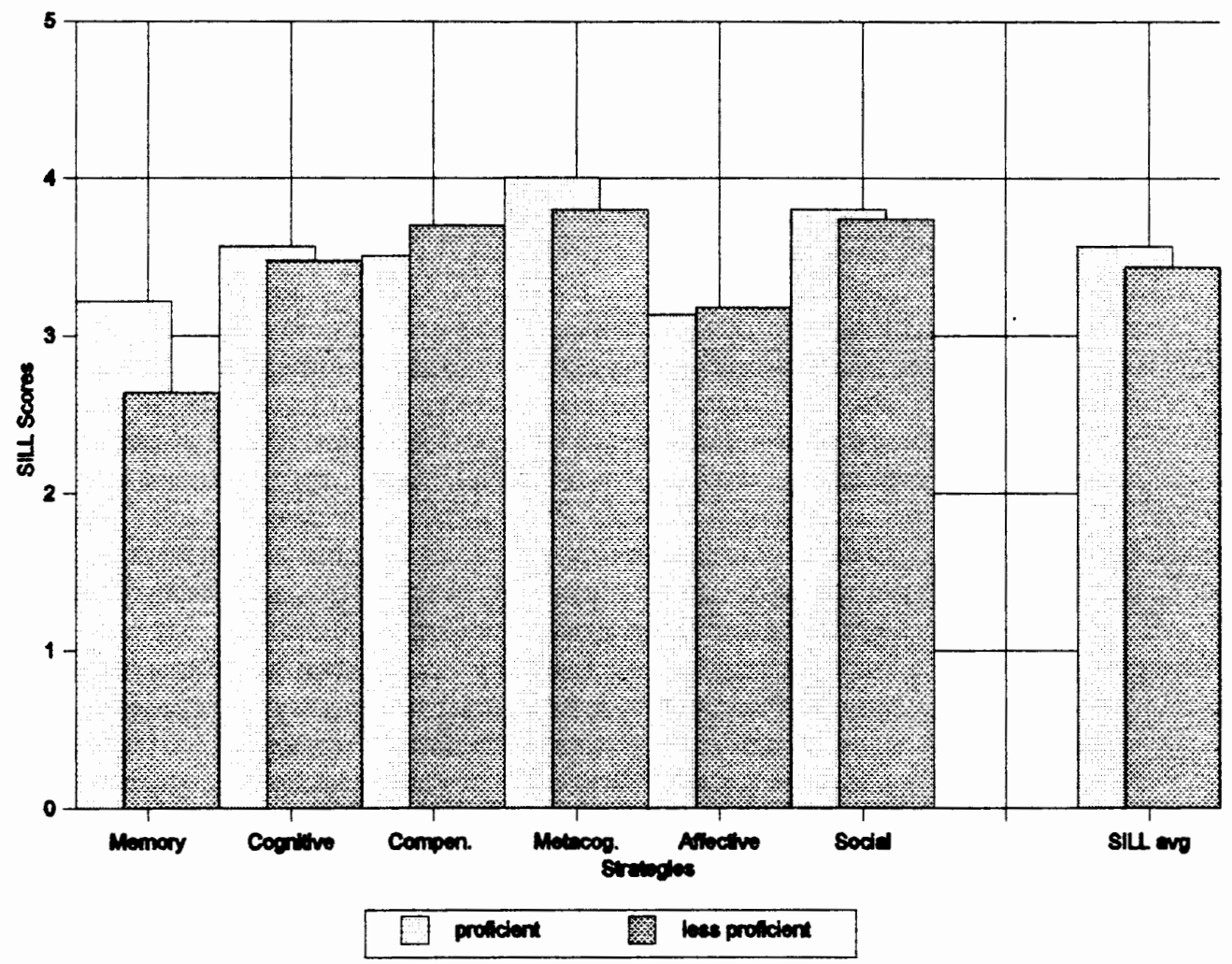

Figure 1. Group means of reported strategy use for the two groups, proficient and less proficient learners, in the six strategy categories of the SILL survey and the means of the overall SILL averages for the two groups. 
From the lack of statistically significant difference between these two groups, it is clear that the level of strategy use reported in the metacognitive and affective categories did not reveal any evidence of a higher or lower level of use by more proficient learners.

In summary the statistical analysis reveals that the only category that shows a significant difference between the two groups is the memory strategy, with PLs generally reporting a higher level of use. There are some non significant differences reported in other categories, with PLs and LPLs.

\section{Research Question Four}

The fourth research question asks whether proficient ESL students will use combinations of learning strategies on specific tasks that are different than those used by less proficient learners.

The subjects were chosen because, while their reported use of strategies on the SILL was similar, their proficiency scores were not. The proficient learner had a score of $77.3 \%$, the LPL had $66.6 \%$ on the proficiency measure, a weighted score of their combined Michigan Test and CELT grades (described in Chapter III). Their SILL profile is shown in Table II below. Both reported low use, less than three, on memory and affective use. Their reported use of cognitive and social strategies was similar. Only in the compensation and metacognitive strategy groups did the two report very much difference. However, in these two strategy groups both reported on the SILL that their use of these strategies was at least a three, "somewhat true of me", or higher. 
The number of strategies used on the three different tasks that the two subjects completed in the Think-Aloud Protocol (TAP) and the total number of strategies used are displayed in Table II, along with the SILL averages for each of the subjects.

\section{TABLE II}

\section{STRATEGY USE IN THINK-ALOUD TASKS}

\section{Proficient Learner Less Proficient Learner}

Task

Strategy \#1 \#2 \#3 total

Memory

$\begin{array}{llll}0 & 15 & 0 & 15\end{array}$

Cognitive

$\begin{array}{llll}20 & 7 & 4 & 31\end{array}$

Compen.

$\begin{array}{lll}2 & 0 & 6\end{array}$

Metacog.

$\begin{array}{lll}3 & 4 & 11\end{array}$

Task

$\# 1 \# 2 \quad \# 3$ total

$\begin{array}{llll}0 & 8 & 0 & 8\end{array}$

$\begin{array}{llll}23 & 18 & 9 & 50\end{array}$

$\begin{array}{llll}2 & 0 & 2 & 4\end{array}$

$\begin{array}{llll}1 & 5 & 7 & 13\end{array}$

Affective

$15 \quad 15 \quad 38$

\begin{tabular}{llll}
5 & 8 & 11 & 24 \\
\hline
\end{tabular}

\begin{tabular}{l}
$45 \quad 49 \quad 70 \quad 194$ \\
\hline
\end{tabular}

Total

$\mathrm{PL}=$ Proficient Learner $\quad \mathrm{LPL}=$ Less Proficient Learner

Compen.$=$ Compensation $\quad$ Metacog. $=$ Metacognitive

Task \# 1 = verb tenses $\quad$ Task \# 2 = word list $\quad$ Task \# 3 = composition

Comparison of SILL scores and strategy use on tasks.

Comparison of the level of strategies the subjects reported using on 
the SILL and their actual strategy use on the tasks shows that there are some differences. Both reported low use of memory strategies (PL 2.9: LPL 2.8). However, the proficient learner, when faced with a task that required use of strategies from this group, efficiently used far more of them than the less proficient learner (task \#2: PL 15: LPL 8). Use of cognitive strategies, as reported on the SILL (PL 3.9: LPL 3.5), fairly well reflected actual use on task one, where these were the appropriate strategies (PL 20: LPL 23). On task three, compensation strategies were some of the most useful. Although the less proficient learner reported higher use on the SILL than the proficient learner (PL 3.0: LPL 4.2), the proficient learner displayed a wider use of strategies from this group on the task (PL 6: LPL 2). Their reported use of metacognitive strategies on the SILL (PL 4.1: LPL 3.3) did reflect their overall use of these strategies (PL 18: LPL 13), and on task three, where metacognitive strategies were particularly useful, the proficient learner used them more (PL 11: LPL 7). Even though they both reported low use of affective strategies on the SILL (PL 2.7: PL 2.7), on task three, where they were expected to be of particular value, the proficient learner employed these strategies far more frequently than the less proficient learner (PL 38: LPL 7). Finally, while both reported high use of social strategies on the SILL (PL 4.3: LPL 4.5), the proficient learner used more strategies from this group on each task and overall use was more than twice as high (PL 24: LPL 11).

Task one.

It was expected that on the first task, filling in the correct verb tense 
in a cloze exercise, that the most appropriate strategies would be those in the cognitive group. Reasoning deductively (applying known grammar rules to new material) and analyzing expressions (examining information in parts) were two cognitive strategies that applied to that task. Both subjects did predominately use this strategy for the task. At 23 uses during the task, the less proficient learner showed a $15 \%$ greater use of cognitive strategies than the proficient learner with 20 instances. While neither subject used many metacognitive strategies (PL 3: LPL 1), the proficient learner did use more. She also had many more instances of affective strategy use (PL 15: LPL 2), seven and a half times more than the less proficient learner.

\section{Task two.}

In task two, which required the subjects to memorize a list of words, the primary strategies that seemed most appropriate for the task were in the memory strategy group. Grouping and associating fall into this category. The proficient learner used nearly twice as many memory strategies as the less proficient learner for this task (PL 15: LPL 8). On the other hand, the less proficient learner, who used a lot of the repeating strategies from the cognitive group, used over two and a half times more cognitive strategies for this task than the proficient learner (PL 7: LPL 18). The two subjects used nearly the same number of metacognitive strategies (PL 4: LPL 5). As in task one, the PL used many more affective strategies for task two than the LPL. Her use of affective strategies at 15 was nearly 
four times as great as the less proficient learner's (4).

\section{Task three.}

This task consisted of writing a police report about an accident and was quite difficult for both subjects. The researcher surmised that the greatest strategy use for the actual writing would fall in the compensation category (adjusting and approximating the message, using circumlocution) and metacognitive (planning, self-monitoring, and identifying the purpose) strategy group. Because of the difficulty, another appropriate strategy group was affective (making positive statements, using laughter, discussing your feelings). The proficient learner used three times as many compensation strategies (PL 6: LPL 2) and 57\% more metacognitive strategies than the less proficient learner (PL 11: LPL 7). However, the biggest gap, once again, was the proficient learner's much higher use of affective strategies (PL 38: LPL 7), nearly five and a half times as frequent as the less proficient learner. The less proficient learner's main strategy for completing this task was to use cognitive strategies. Twenty nine percent of her total reported strategies (31) were cognitive and she used strategies from this group more than twice as often as the proficient learner (PL 4: LPL 9).

\section{Research Question Five}

The last research question asked whether the more proficient learner will use more metacognitive and affective strategies while completing 
specific tasks than the less proficient learner.

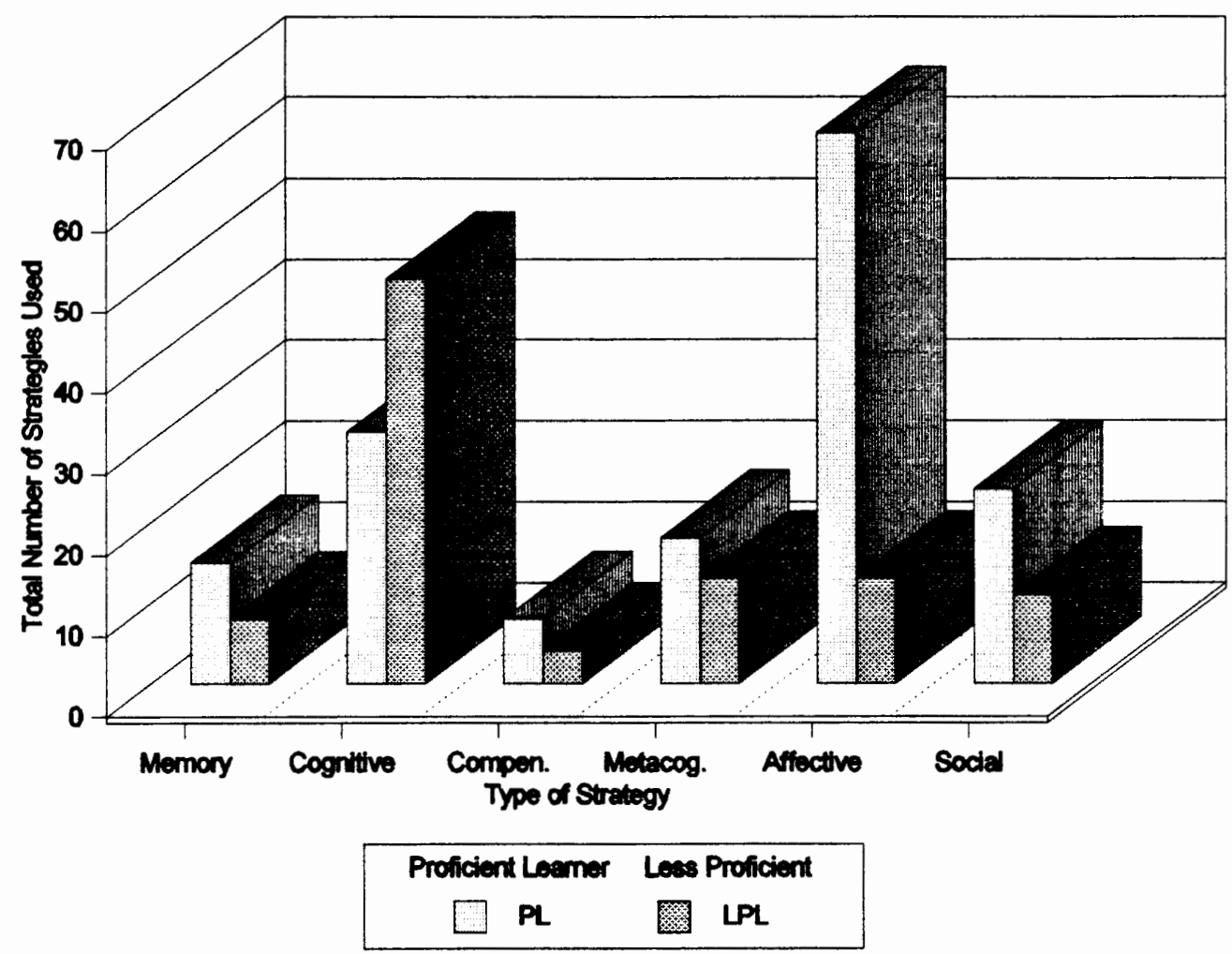

Figure 2. Total number of strategies used by the two subjects in ThinkAloud Protocol for each of the six category groups.

Note. Total number of strategies used by PL on all three tasks $=194$

Total number of strategies used by LPL on all three tasks $=99$

Compen.$=$ Compensation $\quad$ Metacog. $=$ Metacognitive 
As can be seen in Figure two, in all strategy groups except cognitive, the proficient learner demonstrated a far higher level application of strategies. Metacognitive strategies were utilized at a rate $38 \%$ higher than the less proficient learner (PL 18: LPL 13) and affective strategies 5.2 times more frequently (PL 68: LPL 13). In addition, the PL used memory strategies almost twice as often (PL 15: LPL 8), compensation strategies two times more (PL 8: LPL 4), and social strategies over twice as often as the less proficient learner (PL 24: LPL 11). The less proficient learner used strategies from the cognitive group $61 \%$ more frequently than the proficient learner (PL 31: LPL 50).

\section{Overall Strategy Use.}

Social strategies, while not expected to be dominant strategy choices for any of the tasks, were used by both subjects for all three tasks. The two main strategies in this group that were used were asking for clarification and cooperating with proficient users of the language (on these tasks - the researcher). While both learners did use these strategies as they completed each task, the proficient learner used more for each task and, overall, used more than twice as many social strategies for the tasks as the less proficient learner (PL 24: LPL 11). 


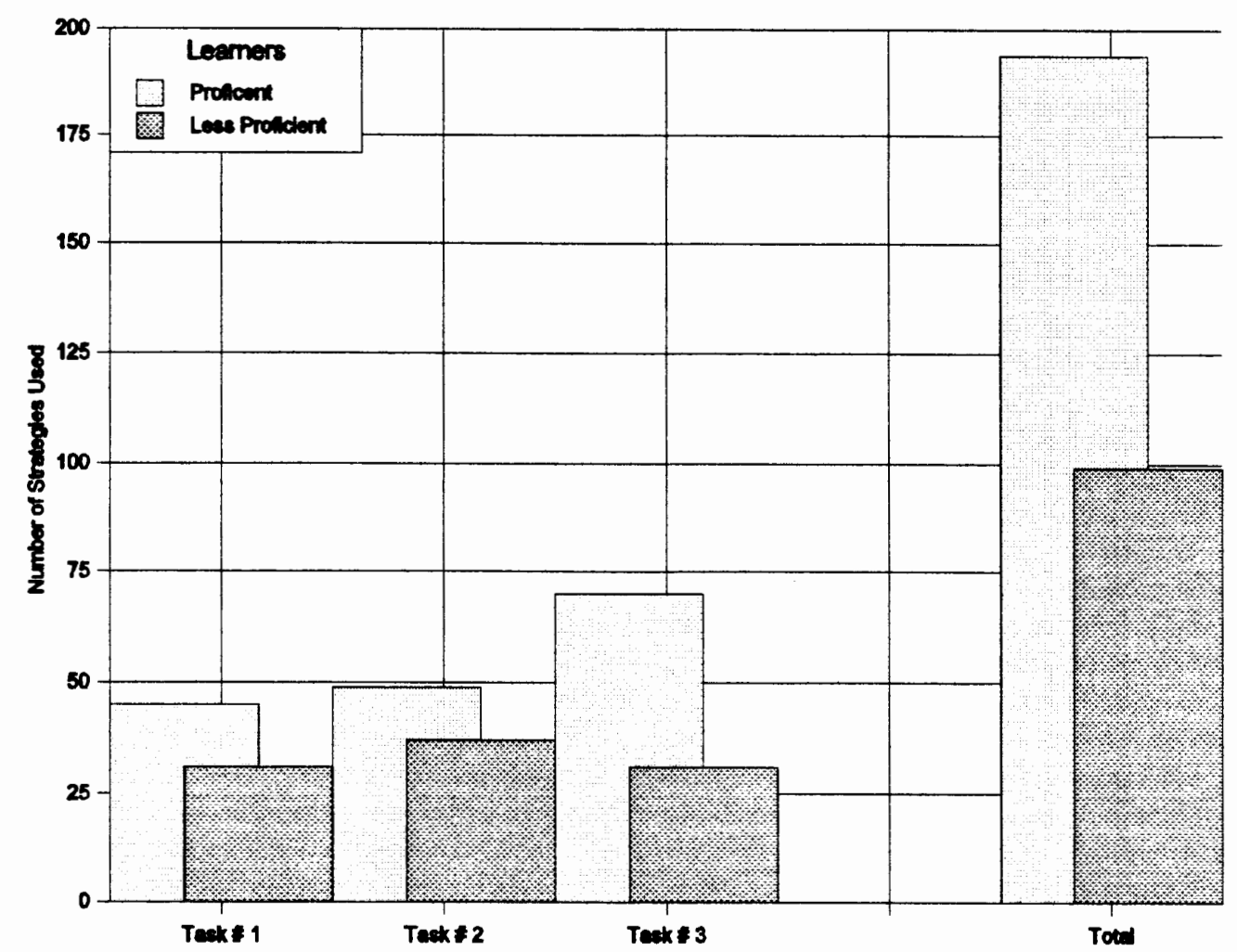

Figure 3. Number of strategies used by the two subjects during each Think-Aloud Protocol task and for the three tasks combined.

In overall strategy use for the combined three tasks, the proficient learner demonstrated a higher use that was nearly twice as great as the less proficient learner (PL 194, LPL 99). Additionally, she used more strategies on each task. For task one the proficient learner used $45 \%$ more strategies than the less proficient learner (PL 45: LPL 31). On task two she used 32\% more (PL 49: LPL 37). On the final task she used 2.2 times as many strategies (PL 70: LPL 31). Figure 3 on the next page reveals the 
substantial difference in the amount of strategies used by the two subjects on each task and overall.

\section{Summary}

To summarize, for task one, where the cognitive strategy group was deemed to be the most appropriate, the LPL demonstrated a higher level of use. Memory, the most effective strategy choice for task two, was implemented more frequently by the proficient learner. In task three, which optimally required the use of compensation, metacognitive and affective strategies, the proficient learner used all three of these strategies more often than the less proficient learner. Finally, on all tasks the proficient learner used more metacognitive strategies and dramatically more affective strategies than the less proficient learner. 


\section{CHAPTER V}

\section{DISCUSSION AND CONCLUSION}

This chapter will discuss the results of the study. It will interpret the findings in the research analyzed in the last chapter as it answers the research questions that were introduced in chapter one. It will also look at similarities and differences in the SILL and the Think-Aloud Protocol (TAP). In the conclusion section, it will also review the similarities and differences of the SILL and Think-Aloud Protocol, look at the limitations of the SILL and the TAP, provide some suggestions for further research using these tools and discuss the implications for teaching.

\section{Discussion}

\section{Research question one.}

This question had two connected parts: when proficient learners (PL) are trying to learn a second language, do they report more overall strategy use in all six of the SILL survey strategy groups than less proficient learners (LPL); and, do PLs report at least a score of three (out of five) in every strategy group, and LPLs report a score of under three? The six category groups are: (1) memory - applying techniques to learning a language that help remembering and recalling, (2) cognitive - using the mental processes, (3) compensation - making up for missing knowledge, (4) metacognitive - organizing and managing learning, (5) affective - 
managing emotions, and (6) social - learning with others.

The only significant difference was in the memory strategy category, where PLs did report using statistically significant more strategies than the LPL group. In this category, LPLs had a mean score of less than three (2.64). It is the only mean score in either group that was under three. There were some non significant differences in the other five categories, with the PLs reporting higher strategy use in the categories of cognitive, metacognitive and social and LPLs reporting higher use in the compensation and affective categories. Also, the PL group did have a higher score on the total SILL, but not at a significant level. The subjects from this study were from two different levels of ESL classes, upper intermediate and advanced. All of the subjects in the LPL group were from the upper intermediate class.

One of the possible reasons that the LPL group may have made such a strong showing in all the categories except memory is that, prior to taking the SILL, they had been introduced to the idea of learning strategies. The writing teacher of the upper intermediate class spent one class period about two weeks before they took the SILL discussing the usefulness of various strategies and the advantage to being aware of what strategies were used for different activities. This sensitization beforehand may have made the upper intermediate students more conscious of the kinds of strategies available to them and situations in which they used them. It could also have had another effect. After finding out that better strategy use may be a tool of the proficient learner, they may have been 
reluctant to admit, even in an anonymous survey, that they used fewer strategies than proficient learners. As a result, they may have reported using more strategies than they actually do use.

A more plausible interpretation of the data is that these are not necessarily different groups when it comes to effective strategy use. All of the advanced class and seven students from the upper intermediate class make up the PL group. They were placed in this group because they scored higher on the two tests that made up the proficiency measure. The underlying assumption was that higher scores on these tests was an indicator of better use of strategies. The upper intermediate class students may be at a lower level in the ESL program but they may employ strategies just as effectively as the advanced class. In every strategy category, except memory, both groups look the same, or differ only slightly.

Most of the students in both classes are international students who came to the U.S. to learn English, either so that they can attend regular university classes or just to improve their proficiency in the language. While a background interview was not done on all the subjects that took the SILL, it is not unreasonable to deduce that upper intermediate students have been in the U.S. studying English for a shorter period of time. This does have support from two areas: (1) seven out of the twelve students in the upper intermediate class are part of the proficient learner group, and (2) both of the students in the think-aloud protocol study stated in the interview that they had been in the U. S. (and in the ESL program) for only 
nine months, the amount of time it would take for students entering at the beginning level to be completing the upper intermediate level. In this case, the best definition of proficiency may not have been the two standardized tests. Those students who had almost completed the advanced level class should have been able to get higher scores on these two tests, since the results of these tests are used to help decide at what level students should be studying. In other words, the advanced students are expected to score higher on these tests than upper intermediates, who are expected to score higher than the next lower level class, etc. The actual results of the proficiency measure reveal a different picture. The two highest proficiency scores came from the upper intermediate class and the lowest score in the PL group was from the advanced class. Table III shows the ranking of all the students in the PL group according to their proficiency scores.

This table clearly indicates that, at least in this instance, class level alone is not necessarily an indicator of proficiency. The table does not include the LPLs, all from the upper intermediate class. If they were included, they would rank numbers 13 through 17. However, their average SILL scores alone do not present a different profile than the PL $\mathbf{s}$. For numbers 13 through 17 , these were $3.8,3.5,3.2,3.2$ and 3.5 , respectively. The one score, besides their lower proficiency scores, that does distinguish them from the others is that all five of them reported the use of memory strategies at less than three. Only three students in the PL group reported a score of less than three in this category. The only other 
distinguishing feature in the LPL group is that four out of five were from Asian countries. The researcher has taught English to successful Chinese

TABLE III

PROFICIENCY RANKING OF PLs

$\begin{array}{ccc}\text { Student } & \text { Class } & \text { SUL Avg } \\ 1 & \text { Up. Int. } & 3.7 \\ 2 & \text { Up. Int. } & 3.8 \\ 3 & \text { Adv. } & 4.0 \\ 4 & \text { Adv. } & 3.4 \\ 5 & \text { Up. Int. } & 3.1 \\ 6 / 7 \text { (tie) } & \text { Up. Int. \& Adv. } & 3.5 \& 3.9 \\ 8 & \text { Up. Int. } & 3.7 \\ 9 & \text { Up. Int. } & 3.0 \\ 10 / 11 \text { (tie) } & \text { Up. Int. \& Adv. } & 3.4 \& 3.9 \\ 12 & \text { Adv } & 3.4 \\ \text { PL = Proficient Learner } & \text { Adv. = Advanced level students } \\ & \text { Up. Int. = Upper Intermediate Students }\end{array}$

students in Asia and knows that one of the main techniques used to learn the Chinese language (and other class room material) is rote memorization. It may be the difference between successful from less successful Chinese students of English is the capacity to move away from using this technique alone, and finding additional memory strategies to aid them in their 
learning endeavors. If students from other Asian countries also rely primarily on rote memorization, this may be the reason that those in the LPL are advancing more slowly.

Since there was no reported difference in strategy use for the other categories in this study, it might be that the use strategies from all groups at a level of at least three may influence the ability to be more successful with their language learning efforts. The reported lower use of strategies from only one category, in this case memory, may significantly influence learners' success in learning a foreign language.

\section{Research question two.}

This question asked: do proficient learners use more metacogntive and affective strategies than do less proficient learners?

There is no evidence in this part of the study that proficient learners use a greater number metacognitive or affective strategies than do less proficient learners. In fact, while PLs reported more use of metacognitive strategies, LPLs actually reported using more affective strategies than did the PL group. Interpretation of this data can only conclude that LPLs, at the very least, perceive themselves as using high levels of these two strategies. More probably, they do use just as do many of these two strategies as those in the PL group. Part of the explanation, again, may be that the upper intermediate class had been exposed to the idea of the importance of broad strategy use in successful language learning prior to taking the SILL survey. Along with the idea that this may have influenced 
their answers on the strategy inventory, it may also be the case that they had more knowledge on how to interpret the statements on the survey. Because of this, they were able to think of more instances when they actually did use these strategies.

However, possibly the best explanation is that these are students who, except for the use of memory strategies, are all aware of the value of using an array of strategies in their language learning. They are all educated people who have a history of being students. One of the prerequisites for success as a student in any field is being able to figure out the best way to learn. Part of this process is being able to use appropriate strategies for different activities. In their past schooling experience they may have already learned that strategies such as organizing one's learning and managing one's emotions are essential ingredients of achievement.

\section{Research question three.}

Is the difference between the level of metacognitive and affective strategy use by proficient learners and less proficient learners greater than the difference in the level of strategy use reported by the group on the other strategy groups? This study shows that there is no difference. Again, the only thing we can infer from the data is that those placed in the LPL group use metacognitive and affective strategies at the same level as the PL and both groups use them at approximately the same level as they use all the other strategies, with the exception of LPLs' lower use of memory strategies. Again, it may be that all of these students are efficient 
acquirers of knowledge; i.e. they are good learners with good learning techniques. According to the students the researcher has talked to, the ESL program is demanding. Also, most of these students are trying to survive in a foreign culture. Those who do not learn to manage their learning and deal successfully with their emotions (both inside and outside the classroom) probably do not succeed in this program. Those who succeed in the program have learned to use metacognitive and affective strategies to manage both their environment and their learning.

\section{Research question four.}

Do proficient learners use combinations of strategies for specific tasks that are different than those used by less proficient learners on the same task?

A number of researchers (Vann \& Abraham, 1990; Raimes, 1985; Perl, 1979) have shown that there is a difference between what strategies proficient learners and less proficient learners use on specific tasks. The Think-Aloud Protocol (TAP) was the tool used by these researchers to discover exactly what strategies were applied to complete the various tasks. The TAP was also used in this study, with interesting results.

Both the proficient learner and less proficient learner had similar profiles and identical SILL averages, 3.5. However, when it came to appropriate strategy use on specific tasks, the proficient learner, generally, used many more of the more efficient task-related strategies than did the less proficient learner. In task two she used $88 \%$ more memory strategies 
(PL 15: LPL 8), the appropriate task related strategy. In task three the proficient learner used three times more compensation strategies (PL 6: LPL 2), 57\% more metacognitive strategies (PL 11: LPL 7), and 5.4 times more affective strategies (PL 38: LPL 7), the most effective strategies for this task. It was only in task one, where the cognitive strategy group included the most suitable strategies, that the LPL showed a slightly greater use of the correct strategies, $15 \%$ more than the proficient learner (PL 20: LPL 23).

From the data analysis, research question four which asks whether proficient learners use combinations of learning strategies on specific tasks that are different than less proficient learmers, appears to be substantiated. What can be inferred from the data is that a proficient learner selects strategies that fit better the task at hand. While the less proficient learner did use slightly more cognitive strategies for the first task, the most appropriate strategies for that activity, the proficient learner also showed a predominant preference for cognitive strategies for the task. The less proficient learner's higher use of cognitive strategies for task one may have simply reflected the less proficient learner's overall preference for this type of strategy, regardless of the task.

The cognitive strategy group is the only one in which the less proficient learner had a total higher use of strategies than the proficient learner. On each task, the less proficient learner used more cognitive strategies than the proficient learner. On task two, $49 \%$ of the strategies she was noted to use were cognitive. On task three, where she could have 
better applied a host of more effective strategies, $29 \%$ of her total strategy use was in the cognitive strategy group. Overall, a little over $50 \%$ of her total strategy use (50 out of 99) fell in this group. On the SILL survey the less proficient learner reported using compensation strategies at 4.2, (PL 3.0) and metacognitive at 3.3 (LP 4.1). However, it is apparent from her strategy use on the different tasks, that she may not use these strategies when it would most benefit her successful completion of an activity.

\section{Research question five.}

The last research question asks if proficient learners use more metacognitive and affective strategies while completing tasks. In the metacognitive group, the proficient learner used a total of $38 \%$ more of these strategies. Although there were relatively few instances of noted use of this strategy (PL 18: LPL 13), on both tasks one and three, she used considerably more strategies from the metacognitive group. Only on task two did the less proficient learner use more (5 vs 4$)$. But, it is in the affective strategy group that the greatest difference was recorded. The proficient learner used 5.2 times more strategies from this group than did the less proficient learner. Also, she used many more of these strategies on each task than did the less proficient learner.

While data collected on strategy use of only two subjects can not be extrapolated to other language learners, in this study the proficient learner did use more metacognitive and affective strategies while completing tasks. Even though on the SILL both reported only a 2.7 level of use of 
affective strategies, the proficient learner used this strategy many times for all tasks. Thirty five percent of all the strategies used by the proficient learner fell within this group.

While it was not one of the research questions, another matter became clear as the data were examined. Not only did the proficient learner use more of the best suited strategies on specific tasks and more affective and metacognitive strategies on all tasks, her strategy use overall far surpassed that of the less proficient learner. She used twice as many strategies overall (PL 194, LPL 99) and more strategies in every strategy group, with the exception of cognitive strategies.

\section{Comparisons and limitations of the SILL survey and the TAP.}

The two instruments are alike in that they can both collect data on the strategy use of second language learners. However, there are a couple of major differences in the instruments which necessarily dictate what can be expected from each.

One of the major differences is how the data are collected. The SILL only asks students to report what strategies they use, while the TAP allows researchers to observe which strategies are actually being used while actual activities are going on. The difference between these is that the information at the learner's disposal with a self-report, like the SILL, comes from long term memory and may not be as reliable. However, what is collected from the TAP, self-revelation data, is in short term memory and is considered more accurate (Cohen, 1987; Ericsson \& Simon, 1987). 
This study has revealed that there can be a considerable difference between what students report using and what they actually use.

Another major difference is that the SILL can collect information on many subjects. This allows for the use of statistical analysis that can provide validity. Because of the small numbers that have, to date, been used to collect data on the TAP, the interpretation of the researcher is the only analysis that can be done. While it is possible that comparisons between studies can be made using the same definitions of strategies (Raimes, 1985; Perl, 1979), both the lack of agreement among researchers on strategy taxonomies and the necessarily small scale nature of the TAP studies make data analysis somewhat subjective and difficult to compare across studies.

Finally, an advantage of the TAP in this particular study is that it provided the researcher with data on a cross section of the strategies used by the subjects on a variety of activities. By doing three tasks that each required different strategies, the researcher was able to gather data not available from studies where only one task, such as a writing assignment, was used.

\section{Conclusion}

The final section will look at some of the problems the researcher encountered in doing the study and provide suggestions for future researchers. It will conclude by discussing how the findings in the study may help teachers in their teaching of ESL. 


\section{Problems with the SILL encountered in this study.}

The biggest problem with the study was the population size. In this study the total size of the two samples combined was only 17 because the decision to use only those students in the top two levels of an ESL program at one university precluded the use of other subjects. While initially it was anticipated there would be more subjects for the study (the two classes combined included over thirty students), many of the students did not qualify for the study because they did not take both the tests that made up the proficiency measure. Furthermore, there were only five subjects in the LPL group. The reason for the small number in this group resulted from the researcher's decision to define proficient learners as those who had scored at least 70 on the proficiency measures. Even in retrospect, this seems like a logical break point. The scores were fairly evenly distributed between the highest score of 82 and 70 , and then there was a nearly four point drop with the next highest score at 66.6. These two factors, small size of population and small size of the LPL sample, make the interpretation of the data problematic, since most studies of this type ideally should have a population of at least 60 (Brown, 1988). Even though data analysis was done using the Mann-Whitney U - Wilcoxon Rank Sum W test, statistical instruments used to adjust for smaller populations, the results of a study where one of the samples has only five subjects can not be interpreted as demonstrating validity.

One other problem with the population was that it was not 
differentiated enough between successful and unsuccessful learners. All the students in the study were at least relatively successful or they would not be at the upper intermediate and advanced levels in the university's ESL program. Because they all are experienced language learners and successful students in a variety of areas, they have already developed at least an unconscious awareness of the use of many strategies. More revealing evidence of strategy use may have developed if the study had included a better cross section of both proficient and less proficient learners. Including students from community colleges, where the student population includes more immigrants with limited education, may have provided a population more diverse in their success at SLA.

Another problem that may have influenced the data was that one group (upper intermediate) had been sensitized to the idea of the importance of good learning strategy use in SLA and the other group had not. The researcher was unaware of this until just before the survey was given. This group's sensitization may demonstrate the value of raising students' consciousness around the subject of learning strategies. However, in the opinion of the researcher, it would have been better if either both groups had had classroom time to become more familiar with strategies and strategy choice, or neither group had.

Finally, gathering no background information on the subjects taking the SILL may have been a mistake. Information on how long students had been in the country and their reasons for being here, plus the number of years they had been studying English before entering the country could all 
have provided more insights into ESL achievement.

Problems with the Think-Aloud Protocol in the study.

The decision to use the taxonomy of strategies developed for the SILL survey was based on two requirements stressed by Ericsson and Simon (1993)--that encoding decisions should be clear, specific, and based on theory and procedures held by the researcher and the decision on what strategy definitions to use should be made ahead of time. The researcher believed that the SILL would be the best instrument to use for encoding the strategies used in the TAP. It provides a wide range of strategies and it has been found to have validity across different studies. Generally, the SILL worked well for this study. One strategy that probably could be added to the metacognitive group is that of deciding not to do something. This is exactly what one of the subjects mentioned that she did on the memory task. For that task, it appeared to be a wise strategy choice. Confronted with a list of 15 words, and five minutes to memorize them, she simply decided not to bother with three words she did not know.

One problem encountered included the need to interpret the strategies, sometimes in slightly different ways than the strict definitions in Oxford's work (1990). At times, this required that the researcher use some latitude in interpreting what specific strategies were being used. Another researcher might make another interpretation. For example, on the memory task, it was sometimes difficult to tell the difference between 
grouping, a memory strategy, and repeating, a cognitive strategy. Another problem was that it was difficult to apply the SILL strategies directly to the writing task. The SILL definition of reviewing comes under the memory strategy group and stresses how to make sure that knowledge gets embedded in long term memory for later recall. The subjects performed reviewing type activities on the composition task, mainly rereading the whole text over at least two or three times. This was recorded as skimming, a cognitive strategy, but seemed to entail something slightly different. Also, in the SILL, there are no strategies that apply to editing, an important writing skill. While neither of the subjects did edit, other research has suggested that this is an important strategy used frequently by good writing students.

Another problem is the difference between learning and production strategies (Tarone, 1981; Faerch \& Kasper, 1984). Oxford's SILL strategies focus on gaining linguistic competence. The tasks used in the research required production strategies such as planning certain discourse. A taxonomy that provided more production strategies may have been easier to work with and more efficient for interpretation of the data.

Interpretation of what strategies the two subjects were using as they completed the tasks may have been influenced by their personalities and ability to adjust to the think-aloud process. The proficient learner found it difficult at first to think aloud as she was doing the tasks, but during the training session she adjusted quickly and was able to produce verbalizations throughout the three tasks, with only minimal coaching. 
The less proficient learner had far greater difficulty doing this. Throughout the tasks she was repeatedly reminded to think aloud and at different points had to be coached two or three times within seconds, even though in the interview and personal conversations she was always a responsive communicator. The researcher evaluated this not so much as an unwillingness to cooperate, as having difficulty with the process of thinking aloud. At times, she did not even appear to hear the researcher's requests to speak aloud. The resulting data may have left hidden many of the strategies that the less proficient learner employed because of her inability to adjust to the process the TAP demands. Further training of the two subjects, including having the subjects and researcher review and discuss the tapes of the training tasks, may have helped the subjects in their thinking and speaking aloud during the three main tasks.

Finally, the study may have revealed many more strategies if it had included a self-observation component that allowed the subjects to report their language behavior immediately after each task or immediately after the three tasks had been completed. This would have satisfied the requirement that the information being revealed was still in short term memory and allowed the researcher to employ another valuable data collection tool. Evidence of the importance of this idea was provided by the less proficient learner in a short informal conversation with the researcher immediately after the memory task. The less proficient learner revealed that she made the decision to not even bother with three words that she did not know and concentrate on those she did know. Also, she 
said that to recall, she used a semantic mapping strategy, where she related all the words in a certain relationship/picture visually. Neither of these strategies were available from her verbalizations.

\section{Suggestions for future research.}

Some of the following suggestions for future research are the result of problems encountered in this study, while others come from ideas the researcher developed as she became involved in the two parts of the study.

Future research using the SILL survey could be enhanced or expanded by including some of the following:

1. Studying groups that have at least 60 subjects could provide a higher probability of generating results that show validity and can be used by other researchers.

2. Using two different samples, one that is clearly more (or less) proficient than the other, could provide researchers with a better picture of the difference between strategies used by the two groups. This could be facilitated by having the SILL translated into the subjects' native language. Even semi-literate and pre-literate students could partake in the study if the SILL survey were given by a native speaker to the students, orally. With these two expansions, many more groups could be included in research that uses the SILL. 3. Investigating the connections between the SILL profile and success (or failures) in different types of activities, such as verbal 
communication, writing, grammar acquisition, could uncover evidence of more specific links between strategy use and proficiency. For example, in one piece of research on whether the use of phonological practice helped in vocabulary recall (DuBois, 1991), it was discovered that there was a statistically significant relationship between the use of cognitive strategies and test scores. 4. Using sensitization as a treatment tool, whereby students are: (1) given pretreatment assignments, (2) introduced to the strategies available to them and given some practice activities to develop their use of them, and (3) given post treatment activities, this would provide more evidence as to just how awareness and practice influence proficiency.

5. Collecting background data on subjects would allow researchers to investigate possible correlations between the subjects' prior history and strategy use.

6. Using an instrument like the Meyer-Briggs Type Indicator, has provided other researchers with more in-depth understanding of the connection between personality types and strategy use. Further use of this tool in combination with the SILL may increase understanding of how different types of second language learners use various strategies.

While time consuming and, to date, constrained to the investigation of only a few subjects in a study, the Think-Aloud Protocol is proving to 
provide some valuable insights into the strategies students actually use on different tasks as they pursue SLA. The following ideas may help future researchers secure better information that will expand our knowledge in the whole area of strategy use:

1. Including self-observation data in TAP studies could provide a richer understanding by the researcher of what strategies subjects use. Asking the subject immediately after the task, while information is still in short term memory, would allow the subjects to identify what strategies they used that they were unable to verbalize. Playing back the tape and asking subjects what they were thinking or how they were solving the problem could reveal much more information than the think aloud alone.

2. Employing a video, along with audio, would provide clues to nonverbal strategy use. Gestures, posture, facial expressions and other non-verbal information can be recorded and analyzed with videos. 3. Using at least two raters in the decoding process increases the reliability of the interpretation of data. This would be best accomplished by having the raters work independently of each other.

4. Scheduling longer training periods that allow the subjects to practice thinking aloud on a variety of tasks could increase the subjects' ability to use these tasks on the think-aloud. During the training session, reviewing the tapes and getting the students to realize what strategies they were saying out loud could increase 
their ability to verbalize them during the study. Preparation for the TAP could also include pre-training classes on the kinds of strategies that are available to them and which ones work better on specific language learning and production tasks.

5. One part of the study was particularly successful. The room used to collect the data was sound proof and contained excellent audio equipment. The researcher recommends that all TAP data collection be done in this kind of environment.

6. Collecting data in their native language and transcribing it would allow for the inclusion of low level ESL students. This would help broaden the range of subjects available to researchers 7. To date, most of the studies using the TAP have been limited to using only a small number of subjects. Doing a larger study with more subjects and a greater variety of subjects (beginners, intermediate, advanced, successful, less successful, and unsuccessful) could provide a rich supply of data that would allow other researchers to examine and to compare with their own researcher. This would probably be best undertaken by a doctoral dissertation candidate or academic researchers in the field. 8. While the SILL survey list of strategies worked fairly well, development of a strategy taxonomy that addressed both production and learning would provide researchers that use TAPs with a better encoding instrument. 
The two strategies that turned out to be the most interesting in this study were memory and affective strategies. In the SILL part of the study, memory was the only strategy that showed a statistically significant difference between proficient and less proficient learners. Furthermore, in the TAP the proficient learner did use almost twice as many memory strategies as the less proficient learner. Oxford (1990) believes that good use of memory strategies strongly contributes to learners' language acquisition. Affective strategies were the ones which the proficient learner used most in the TAP. Also, in relationship to the less proficient learner, the proficient learner used affective strategies many more times on all tasks. The findings of this study would recommend that future research on the use of these two strategy groups by various kinds of learners might uncover more evidence of their important place in successful SLA.

\section{Implications for teaching.}

The results of this study indicate that students do not make use of all the types of strategies available to them. Even when students are aware of the different strategies and report using them, they may not necessarily apply the best strategy to different learning and production activities. The link between effective strategy use and success in learning a second or foreign language provides teachers with another tool for assisting their learners both in and out of the classroom. There is slight evidence in the study that, even when students are briefly introduced to the idea of strategies and strategy use and become more conscious of them (as the 
upper intermediate class was), they may realize they use more than they thought.

If this is the case then one of the things that teachers can do is provide strategy training that will build awareness of strategies in students and give them practice in using them. Oxford's book on language learning strategies (1990) provides many student activities that teachers can use. The literature suggests that the best way to teach learning strategies is to link it to the course content (Skehan, 1991; O'Malley \& Chamot 1990). This provides relevance to what students are actually studying. Also, students should have explicit explanations of the best use of specific strategies for various tasks and be required to practice and self-monitor their application of the strategies on different tasks. This improves their ability to use strategies outside the training sessions (Brown, 1992).

The importance of memory and affective strategies revealed in the study provides teachers with the reason to put extra effort into showing students ways in which they can use these strategies to positively influence their language learning. Classroom activities could be designed to further the students' competence in the use of strategy techniques in these two categories. Language teachers outside the U.S. might consider concentrating more time on affective strategy use, since these students have not had the pressing need of international students learning in the U.S.(a foreign culture) to develop good affective strategies to manage their emotions.

Even the think-aloud process can be used in the classroom. An 
article by Davey (1983) describes how teachers can use this technique to show students how to identify the strategies they are using, or should be using as they proceed through a reading assignment. A teacher could design a passage that includes the kinds of strategies that help students understand what they are reading. The passage could include the need for using such strategies as predicting, developing images, making analogies by using prior knowledge, and monitoring and correcting one's comprehension. Then the teacher reads the passage and thinks out loud while the students listen to the process she is going through as she tries to understand unfamiliar material. Activities that apply to other strategies, such as writing, conversation, and grammar lessons could be designed by teachers to show their students how to use different types of strategies in a variety of learning and communicating situations.

Finally, there may be some relationship between students from different cultures and strategy use. Teachers could find out more about the students' backgrounds and prior learning experiences. This information could help them to identify the kinds of help students in their classes would most benefit from. For instance, in this study it appears that using a variety of memory strategies beyond rote memorization may not be a way of learning that Asian students are used to. Improving the ability to use more strategies from this group, such as associating/elaborating, grouping and using key words to link new information to what one already knows, may help them advance more quickly in their endeavors to learn English. 


\section{REFERENCES}

Abraham, R. \& Vann, R. (1987). Strategies of two language learners: A case study. In A. Wenden \& J. Rubin (Eds.), Learner strategies in language learning. (pp. 85-102). Englewood, NJ: PrenticeHall, Inc.

Anderson, J. R. (1982). Acquisition of cognitive skills. Psychological Review, 89, 369-406.

Bacon, S. M. \& Finnemann, M. D. (1990). A study of the attitudes, motives and strategies of university foreign language students and their disposition to authentic oral and written output. Modern Language Journal, 74, 459-473.

Bialystok, E. (1981). The role of conscious strategies in second language proficiency. Modern Language Journal, 65, (24-35).

Brown, A. L. \& Palinscar, A. S. (1982). Inducing strategic learning from texts by means of informed, self-control training. Topics in Learning and Learning Disabilities, 2, 1-17.

Brown, H. D. (1991). Breaking the language barrier: Creating your own pathway to success. Yarmouth, ME: Intercultural Press, Inc.

Brown, H. D. (1987). Principles of language learning and teaching. 2nd ed. Englewood Cliffs, NJ: Prentice-Hall.

Brown, J. D. (1988). Understanding research in second language learming: A teacher's guide to statistics and research design. New York, NY: Cambridge University Press. 
Cavalcanti, M. C. (1987). Investigating FL reading performance through pause protocols. In C. Faerch \& G. Kasper (Eds.), Introspection in second language research (pp. 230-250). Philadelphia, PA:

Multilingual Matters Ltd.

Chamot, A. U. (1987). The learning strategies of ESL student. In A. Wenden \& J. Rubin (Eds.), Learning strategies in language learning (pp. 71-83). Englewood Cliffs, NJ: Prentice Hall, Inc.

Chamot, A. U. \& Kupper, L. (1989). Leaning strategies in foreign language instruction. Foreign Language Annals, 22, 13-24.

Cohen, A. (1995, March). Verbal reports as a source of insights on learner strategies. Paper presented at the colloquium on issues in the assessment of $\mathrm{L} 2$ learner strategies, AAAL annual conference, Long Beach, CA.

Cohen, A. (1990). Language learning: Insights for learners, teachers, and researchers. New York: Newbury House Publishers.

Cohen, A. (1987). Using verbal reports in research on language learning. In C. Faerch \& G. Kasper (Eds.), Introspection in second language research. (pp. 82-95). Philadelphia, PA: Multilingual Matters Ltd.

Davey, B. (1983). Think-aloud - modeling the cognitive processes of reading comprehension. Journal of Reading, 27, 44-47.

Dechert, H. W. (1987). Analyzing language processing through verbal protocols. In C. Faerch \& G. Kasper (Eds.), Introspection in second language research. (pp. 96-112). Philadelphia, PA: Multilingual Matters Ltd.

DuBois, K. A. (1991). The importance of phonological practice and other strategies for vocabulary recall and comprehension (Master's thesis, Portland State University, 1991). 
Ehrman, M. E. (1994). Weakest and strongest learners in intensive language training: A study of extremes. (in press). In C. Klee (Ed.), Faces in a crowd: Individual leamers in multisection programs. Boston, MA: Reinle and Reinle.

Ehrman, M. \& Oxford, R. (1995). Cognition plus: Correlates of language learning success. Modern Language Journal, 79, 67-89.

Ehrman, M. \& Oxford, R. (1990). Adult language learning styles and strategies in an intensive training setting. Modem Language Journal, 74. 311-327.

Ehrman, M. \& Oxford, R. (1989). Effects of sex differences, career choice, and psychological type on adult language learning strategies. The Modern Language Journal. 73, 1-13.

Ely, C. (1988). Personality: Its impact on attitudes toward classroom activities. Foreign Language Annals, 21, 25-32.

Ericsson, K. A. \& Simon, H. A. (1993). Protocol analysis: Verbal reports as data (revised edition). Cambridge, MA: The MIT Press.

Ericsson, K. A. \& Simon, H. A. (1987). Verbal reports on thinking. In C. Faerch \& G. Kasper (Eds.), Introspection in second language research. (pp. 24-53). Philadelphia, PA: Multilingual Matters Ltd.

Ericsson, K. A. \& Simon, H. A. (1984). Protocol Analysis: Verbal reports as data. Cambridge, MA: The MIT Press

Faerch, C. \& Kasper, G. (1984). Two ways of defining communication strategies. Language Learning, 34, 45-63.

Gagne, E. D., Yekovich, C. W. \& Yekovich, F. R. (1993). The cognitive psychology of school learning (second edition). New York, $\mathrm{N}$. Y.: Harpers Collins College Publishers. 
Haastrup, K. (1987). Using thinking aloud and retrospection to uncover learners' lexical inferencing procedures. In C. Faerch \& G. Kasper (Eds.), Introspection in second language research. (pp. 197-212). Philadelphia, PA: Multilingual Matters Ltd.

Hofstede, G. (1991). Cultures and organizations: Software of the mind. Berkshire, England: McGraw-Hill Book Company Europe.

Horwitz, E. K., Horwitz, M. B. \& Cope, J. A. (1986). Foreign language classroom anxiety. Modern Language Journal, 70, 125-132.

Krashen, S. (1982). Principles and practice in second language acquisition. Elmsford, New York: Pergamon Press, Inc.

Krings, H. P. (1987). The use of introspective data in translation. In C. Faerch \& G. Kasper (Eds.), Introspection in second language research. (pp. 159-176). Philadelphia, PA: Multilingual Matters Ltd.

Kumaravadivelu, B. (1994). The postmethod condition: Emerging strategies for second/foreign language teaching. TESOL, 28, 27-48.

Larsen-Freeman, D. (1991). Second language acquisition: Staking out the territory. TESOL Quarterly, 25, 315-339.

Lachman, R., Lachman, J. L. \& Butterfield, E. C. (1979). Cognitive psychology and information processing. Hillsdale, N. J.: Erlbaum.

Lukmani, Y. M. (1972). Motivation to learn and language proficiency. Language Learning, 22, 161-273.

Madsen, H. (1990). In D. Douglas (Ed.), Standardized ESL tests used in U.S. colleges and universities (pp. 27-40). Washington DC: National Association for Foreign Student Affairs.

McKay, S., Rosenthal, L. (1980). Writing for a specific purpose. Englewood Cliffs, NJ: Prentice Hall. 
Nayak, N., Hansen, N. , Krueger, N. \& McLaughlin, B. (1990). Language-learning strategies in monolingual and multilingual adults. Language Learming. 40, 221-244.

Nyikos, M. \& Oxford, R. (1993). A factor analytic study of language-learning strategy use: Interpretation from information processing theory and social psychology. The Modern Language Journal, 77 11-21.

Nyikos, M. (1990). Sex-related differences in adult language learning: Socialization and memory factors. The Modern Lanquage Journal, 74, 273-287.

O'Malley, J. M. \& Chamot, A. U. (1990)Leaming strategies in second language acquisition. New York: Cambridge University Press

O'Malley, J. M., Chamot, A. U., Stewner-Manzanares, G., Kupper, L., Russo, R. P. (1985). Learning strategies used by beginning and intermediate ESL students. Language Learning, 35, 21-46.

Oxford, R. L. (1990). Language learning strategies: What every teacher should know. New York: Newbury House Publishers.

Oxford, R. L. (1990b). Missing link: Evidence from research on language learning styles and strategies. Georgetown University Round Table on Languages and Linguistics 439-458.

Oxford, R. L. (1993). Research on second language learning strategies. Annual Review of Applied Linguistics, 13, 175-187.

Oxford, R. \& Crookall, D. (1989). Research on language learning strategies: Methods, findings, and instructional issues. The Modern Language Journal, 73, 404-419.

Oxford, R., Crookall, D., Cohen, A., Lavine, R., Nyikos, M., Sutter, W. (1990). Strategy training for language learners: Six situational case studies and a training model. Foreign Language Annals, 22, 197-216. 
Oxford, R. \& Ehrman, M. (1993). Second language research on individual differences. Annual Review of Applied Linguistics, 13 188205.

Oxford, R., Lavine, R., Crookall, D. (1989). Language learning strategies, The communicative approach and their classroom implications. Foreign Language Annals, 22, 29-39.

Oxford, R. \& Nyikos, M. (1989). The variables affecting choice of language learning strategies in university students. The Modern Language Journal. 73, 291-300.

Oxford, R, Young P., Ito, S., \& Sumrall, M. (1993). Learning a language by satellite television: What influences student achievement? System, 21, 31-48.

Pearson, E. (1988). Learner strategies and learner interviews. ELT Journal, 42 , 172-178.

Perl, S. (1979). The composing processes of unskilled college writers. Research in the Teaching of English, 13, 317-336.

Raimes, A. (1987). Language proficiency, writing ability, and composing strategies: A study of ESL college student writers. Language Learning. 37 , 439-467.

Raimes, A. (1985). What unskilled ESL's students do as they write: A classroom study of composing. TESOL Quarterly, 19 229-258.

Reid, J. M. (1987). The learning style preferences of ESL students. IESOL Quarterly. 21, 87-109.

Reiss. M. (1981). Helping the unsuccessful learner. Modern Language Journal, 65, 122-127. 
Richards, C., Platt. J. \& Platt, H. (1992). Longman dictionary of language teaching \& applied linguistics. Essex, England: Longman House.

Rubin, J. (1975). What the "good language learner" can teach us. TESOL Quarterly, 9, 41-51.

Rubin, J. (1987). Learning strategies: Theoretical assumptions, research history and typology. In A. Wenden \& J. Rubin (Eds.), Learner strategies in language learning (pp. 15-30). Englewood, NJ: PrenticeHall, Inc.

Skehan, P. (1991). Individual differences in second language learning. Studies in Language Acquisition, 13, 275-297.

Smagorinsky, P. (1994). Think-aloud protocol analysis: Beyond the black box. In P. Smagorinsky (Ed.), Speaking about writing (pp. 319). Thousand Oaks, CA: Sage Publications.

Sparks, R. L. \& Granschow, L. (1991). Foreign language learning differences: Affective or native language aptitude differences. The Modern Language Journal, 75, 3-11.

Spolsky, B. (1988). Bridging the gap: A general theory of second language learning. TESOL Quarterly, 22, 377-396.

Spolsky, B. (1985). Formulating a theory of second language learning. Studies in Second Language Acquisition, 7, 269-288.

Tarone, E. (1981). Some thoughts on the notion of communication strategy. TESOL Quarterly, 15, 285-295.

Vann, R. \& Abraham, R. (1990). Strategies of unsuccessful language learners. TESOL Journal, 24, 177-199. 
Waters, M. \& Waters, A. (1992). Study skills and study competence: Getting the priorities right. ELT Journal, 46, 264-273.

Witte, S. P. \& Cherry, R. D. (1994). Think-aloud protocols, protocol analysis, and research design: An explanation of the influences of writing tasks on writing processes. In P. Smagorinsky (Ed.), Speaking about writing. (pp. 20-54). Thousand Oaks, CA: Sage Publications.

Wittrock, M. C. (1983). Writing and the teaching of reading. Language Arts, 60, 600-606.

Wittrock, M. C., Marks, C. B., \& Doctorow, M. J. (1975). Reading as a generative process. Journal of Educational Psychology, 67 484-489.

Young, D. J. (1991). Creating a low-anxiety classroom environment: What does language anxiety research suggests. The Modern Language Journal, 75, 426-439. 
APPENDIX A

STRATEGY INVENTORY FOR LANGUAGE LEARNING (SILL)

SURVEY 
STRATEGY INVENTORY FOR LANGUAGE LEARNING (SILL)

\section{Version for Speakers of Other Languages Learning English Version 7.0 (ESL/EFL)}

(c) R. Oxford 1989 (Oxford, 1990, 293-296)

Directions

This form of the of the STRATEGY INVENTORY FOR LANGUAGE LEARNING (SILL) is for students of English as a second or foreign language. You will find statements about learning English. Please read each statement. On the separate Worksheet, write the response $(1,2$, 3,4 , or 5) that tells HOW TRUE OF YOU THE STATEMENT IS.

1. Never or almost never true of me

2. Usually not true of me

3. Somewhat true of me

4. Usually true of me

5. Always or almost always true of me

NEVER OR ALMOST NEVER TRUE OF ME means that the statement is yery rarely true of you.

USUALLY NOT TRUE OF ME means that the statement is true less than half the time.

SOMEWHAT TRUE OF ME means that the statement is true of you about half the time.

USUALLY TRUE OF ME means that the statement is true more than half the time.

ALWAYS OR ALMOST ALWAYS TRUE OF ME means that the statement is true of you almost always.

Answer $\mathrm{n}$ terms of how well the statement describes you. Do not answer how you think you should be, or what other people do. There are not right or wrong answers to these statements. Put your answers on the separate 
Worksheet. Please make no marks on the items. Work as quickly as you can without being careless. This usually takes about 20-30 minutes to complete. If you have any questions, let the teacher know immediately.

\section{EXAMPLE}

Read the item, and choose a response ( 1 through 5 above), and write it in the space after the item.

English.

I actively seek out opportunities to talk with native speakers of

You have just completed the example item. Answer the rest of the items on the Worksheet.

\section{Part A}

1. I think of relationships between what I already know and new things I learn in English.

2. I use new English words in a sentence so I can remember them.

3. I connect the sound of a new English word and an image or picture of the word to help me remember the word.

4. I remember a new English word by making a mental picture of a situation in which the word might be used.

5. I use rhymes to remember new English words.

6. I use flashcards to remember new English words.

7. I physically act out new English words.

8. I review English lessons often. 
9. I remember new English words or phrases by remembering their location on the page, on the board, or on a street sign.

\section{Part B}

10. I say or write new English words several times.

11. I try to talk like native speakers.

12. I practice the sounds of English.

13. I use the English words I know in different ways.

14. I start conversations in English.

15. I watch English language TV shows spoken in English or go to movies spoken in English.

16. I read for pleasure in English.

17. I write notes, messages, letters, or reports in English.

18. I first skim an English passage (read over the passage quickly) then go back and read carefully.

19. I look for words in my own language that are similar to new words in English.

20. I try to find patterns in English.

21. I find the meaning of an English word by dividing it into parts that I understand.

22. I try not to translate word-for-word.

23. I make summaries of information that I hear or read in English. 


\section{Part C}

24. To understand unfamiliar English words, I make guesses.

25. When I can't think of a word during a conversation in English, I use gestures.

26. I make up new words if I do not know the right ones in English.

27. I read English without looking up every new word.

28. I try to guess what the other person will say next in English.

29. If I can't think of an English word, I use a word or phrase that means the same thing.

\section{Part D}

30. I try to find as many ways as I can to use my English.

31. I notice my English mistakes and use that information to help me do better.

32. I pay attention when someone is speaking English.

33. I try to find out how to be a better learner of English

34. I plan my schedule so I will have enough time to study English.

35. I look for people I can talk to in English.

36. I look for opportunities to read as much as possible in English.

37. I have clear goals for improving my English skills.

38. I think about my progress in learning English. 


\section{Part E}

39. I try to relax whenever I feel afraid of using English.

40. I encourage myself to speak English even when I am afraid of making a mistake.

41. I give myself a reward or treat when I do well in English.

42. I notice if I am tense or nervous when I am studying or using English.

43. I write down my feelings in a language learning diary.

44. I talk to someone else about how I feel when I am learning English.

\section{Part $F$}

45. If I do not understand something in English, I ask the other person to slow down or say it again.

46. I ask English speakers to correct me when I talk.

47. I practice English with other students.

48. I ask for help from English speakers.

49. I ask questions in English.

50. I try to learn about the culture of English speakers. 
Your Name Date

\section{Worksheet for Answering and Scoring}

The Strategy Inventory for Language Learning (SILL)

Version 7.0 (ESL/EFL)

(c) R. Oxford, 1989 (Oxford, 1990, 297-298)

1. The blanks (_ ) are numbered for each item on the SILL.

2. Write your response to each item (that is, write $1,2,3,4$, or 5) in each of the blanks.

3. Add up each column. Put the results on the line marked SUM.

4. Divide by the number under SUM to get the average for each column. Round this average off to the nearest tenth, as it 3.4.

5. Figure out your overall average. To do this, add up all the SUMS for the different parts of the SILL. Then divide by 50 .

6. When you have finished, your teacher will give you the Profile of Results. Copy your averages (for each part and for the whole SILL) from the Worksheet to the Profile. 
SILL Worksheet

Version 7.0 (ESL/EFL)

PartA Part B PartC PartD PartE PartE Whole SILL 1._ 10_ 24_ 30_ 39_ 45. SUM Part A

2. 11- 25- 31- 40- 46- SUM Part B

3. 12- 26. 32. 41- 47- SUM Part C

4. 13. 27- 33- 42. 48- SUM Part D

5- 14- 28- 34- 43- 49- SUM Part E-

6. $15-29-35-44-50-$ SUM Part F-

8. 17. 37 .

9. 18- 38 -

19.

20.

21.

22.

23.

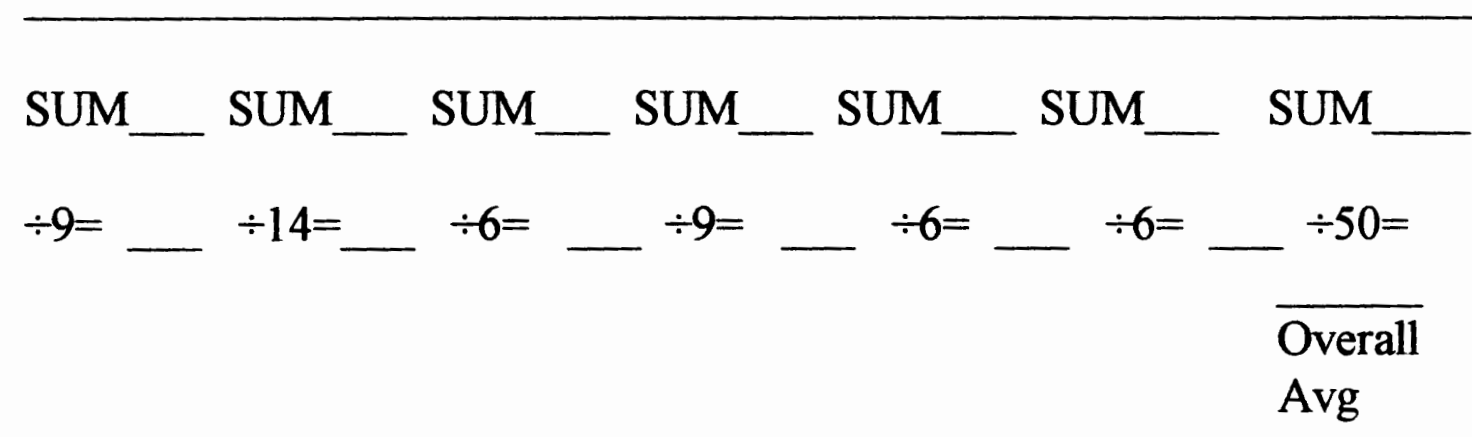

Your Name

Date 
Profile of Results on the Strategy Inventory for Language Learning (SILL)

$$
\text { Version } 7.0
$$

(c) R. Oxford, 1989 (Oxford, 1990, 299)

You will receive this Profile after you have competed the Worksheet. This Profile will show your SILL results. These results will tell you the kinds of strategies you use in learning English. There are no right or wrong answers.

To complete this profile, transfer your averages for each part of the SILL, and your overall average for the whole SILL. These averages are found on the Worksheet.

Part A What Strategies Are Covered Your Average on This Part
A.
Remembering more effectively
B. Using all your mental processes
C. Compensating for missing knowledge
D. Organizing and evaluating your learning
E. Managing your emotions
F. Learning with others

YOUR OVERALL AVERAGE 
APPENDIX B

DEFINITIONS OF LEARNING STRATEGIES

USED IN THE SILL 
DEFINITIONS OF STRATEGIES IN THE

STRATEGY INVENTORY FOR LANGUAGE LEARNING (SILL)

(Source: Oxford, 1990)

The following definitions of the six strategy categories used in the study and the strategies that make up each category are taken verbatim from Oxford's book: Language Learning Strategies.

\section{Memory Strategies}

Memory strategies reflect very simple principles, such as arranging things in order, making associations, and reviewing. These principles all involve 'meaning'. For the purpose of learning a new language, the arrangement and associations must be personally meaningful to the learner, and the material to be reviewed must have significance.

\section{Creating Mental Linkages}

In this set are three strategies that form the cornerstone for the rest of the memory strategies; grouping, associating/elaborating, and using context.

\section{Grouping.}

Classifying or reclassifying language material into meaningful units, either mentally or in writing, to make the material easier to remember by reducing the number of discrete elements. Groups can be based on type of word (e.g., all nouns or verbs), topic (e.g. . words about weather), practical functions (e.g., terms for things that make a car work), linguistic function (e.g., apology, request, demand), similarity (e.g., warm, hot, tepid, tropical), dissimilarity or opposition (e.g., friendly/unfriendly), the way one feels about something (e.g., like, dislike), and so on. The power of this strategy may be enhanced by labeling the groups, using acronyms to remember groups, or using different colors to represent different groups. 


\section{Associating/Elaborating.}

Relating new language information to concepts already in memory or relating one piece of information to another, to create associations in memory. These associations can be simple or complex, mundane or strange, but they must be meaningful to the learner. Associations can be between two things, such as bread and butter, or they can be in the form of a multipart "development." such as school-book-paper-tree-country-earth. They can also be part of a network, such as semantic mapping (see below).

\section{Placing new words into context.}

Placing a word or phrase in a meaningful sentence, conversation, or story in order to remember it. This strategy involves a form of associating/elaborating, in which the new information is linked with a context. This strategy is not the same as guessing intelligently, a set of compensation strategies (described later) which involve using all possible clues, including the context, to guess the meaning.

\section{Applying Images and Sounds}

Four strategies are included here: using imagery, using keywords, semantic mapping and representing sounds in memory. These all involve remembering by means of visual images or sounds.

\section{Using imagery}

Relating new language information to concepts in memory by means of meaningful visual imagery, either in the mind or in actual drawing. The image can be a picture of an object, a set of locations for remembering a sequence of words or expressions, or a mental representation of the letters of a word. This strategy can be used to remember abstract words by associating such words with a visual symbol or a picture of a concrete object.

\section{Semantic mapping.}

Making an arrangement of words into a picture, which has a key concept at the center or at the top, and related words and concepts linked with the key concept by means of lines or arrows. This strategy involves meaningful imagery, grouping, and associations; it visually shows how 
certain groups of words relate to each other.

\section{Using keywords.}

Remembering a new word by using auditory and visual links. The first step is to identify a familiar word in one's own language that sounds like the new word - this is the "auditory link." The second step is to generate an image of some relationship between the new word and a familiar one -- this is the "visual link." Both links must be meaningful to the learner. For example, to learn the new French word potage (soup), the English speaker associates it with a pot and then pictures of a pot full of potage. To use a keyword to remember something abstract, such as a name, associate it with a picture of something concrete that sounds like the new word. For example, Minnesota can be remembered by the image of a mini-soda.

\section{Representing sounds in memory}

Remembering new language information according to sound. This is a broad strategy that can use any number of techniques, all of which create a meaningful, sound-based association between the new material and already know material. For instance, you can (a) link a target language word with any other word (in any language) that sounds like the target language word, such as Russian brat (brother) and English brat (annoying person), (b) use phonetic spelling and/or accent marks, or (c) use rhymes to remember a word.

\section{Reviewing Well}

This category contains study one strategy, structured reviewing. Looking at new target language information once is not enough; it must be reviewed in order to be remembered.

\section{Structured reviewing}

Reviewing in carefully spaced intervals, at first close together and then more widely spaced apart. This strategy might start, for example, with a review 10 minutes after the initial learning, then 20 minutes later, an hour or two later, a day later, 2 days later, a week later, and so on. This is sometimes called "spiraling," because the learner keeps spiraling back to 
what was already been learned at the same time that he or she is learning new information. The goal is "overlearning" -- that is, being so familiar with information that it becomes natural and automatic.

\section{Employing_Action}

The two strategies in this set, using physical response or sensation and using mechanical tricks, both involve some kind of meaningful movement or action. These strategies will appeal to learners who enjoy the kinesthetic or tactile modes of learning.

\section{Using physical response or sensation.}

Physically acting out new expressions (e.g., going to the door), or meaningfully relating a new expression to a physical feeling or sensation (e.g., warmth).

2. Using mechanical techniques.

Using creative but tangible techniques, especially involving moving or changing something which is concrete, in order to remember new target language information. Examples are writing words on cards and moving cards from one stack to another when a word is learned, and putting different types of material in separate sections of a language learning notebook.

\section{Cognitive Strategies}

Cognitive strategies are essential in learning a new language. Such strategies are a varied lot, ranging from repeating to analyzing expressions to summarizing. With all their variety, cognitive strategies are unified by a common function: manipulation or transformation of the target language by the learner. Cognitive strategies are typically found to be the most popular strategies with language learners.

\section{Practicing}

Of the five practicing strategies, probably the most significant one is practicing naturalistically. 


\section{Repeating.}

Saying or doing something over and over: listening to something several times; rehearsing; imitating a native speaker.

2. Formally practicing with sounds and writing systems.

Practicing sounds (pronunciation, intonation, register, etc.) in a variety of ways, but not yet in naturalistic communicative practice; or practicing a new writing system of the target language.

\section{Recognizing and using formulas and patterns.}

Being aware of and/or using routine formulas (single, unanalyzed units), such as "Hello, how are you?"; and unanalyzed patterns (which have at least one slot to be filled), such as, "It's time to

\section{Recombining.}

Combining known elements in a new way to produce a longer sequence, as in linking one phrase with another in a whole sentence.

\section{Practicing naturalistically.}

Practicing the new language in natural, realistic settings, as in participating in a conversation, reading a book or article, listening to a lecture, or writing a letter in the new language.

\section{Receiving and Sending Messages}

Two strategies for receiving and sending messages are (a) getting the idea quickly and (b) using resources for receiving and sending messages. The former uses two specific techniques for extracting ideas, while the latter involves using a variety of resources for understanding or producing meaning.

\section{Getting the idea quickly.}

Using skimming to determine the main ideas or scanning to find specific details of interest. This strategy helps learners understand rapidly what they hear or read in the new language. Preview questions often assist. 
2. Using resources for receiving and sending messages.

Using print or nonprint resources to understand incoming messages or produce outgoing messages.

Analyzing and Reasoning

This set of five strategies concerns logical analysis and reasoning as applied to various target language skills. Often learners can use these strategies to understand the meaning of a new expression or to create a new expression.

1. Reasoning deductively

Using general rules and applying them to new target language situations. This is a top-down strategy leading from general to specific.

2. Analyzing expressions.

Determining the meaning of a new expression by breaking it down into parts; using the meanings of various parts to understand the meaning of the whole expression.

\section{Analyzing contrastively}

Comparing elements (sounds, vocabulary, grammar) of the new language with elements of one's own language to determine similarities and differences.

\section{Translating.}

Converting a target language expression into the native language (at various levels, from words and phrases all the way up to whole texts); or converting the native language into the target language; using one language as the basis for understanding or producing the other.

\section{Transferring}

Directly applying knowledge of words, concepts, or structures from one language to another in order to understand or produce an expression in the new language. 


\section{Creating Structure for Input and Output}

The following three strategies are ways to create structure, which is necessary for both comprehension and production in the new language.

\section{Taking notes.}

Writing down the main idea or specific points. This strategy can involve raw notes, or it can comprise a more systematic form of notetaking such as the shopping list format, the T-formation, the semantic map, or the standard outline form.

\section{Summarizing.}

Making a summary or abstract of a longer passage.

\section{Highlighting.}

Using a variety of emphasis techniques (such as underlining, starring, or color-coding) to focus on important information in a passage.

\section{Compensation Strategies}

Compensation strategies enable learners to use the new language for either comprehension or production despite limitations in knowledge. Compensation strategies are intended to make up for an inadequate repertoire of grammar and, especially, of vocabulary.

\section{Guessing Intelligently in Listening and Reading}

The two strategies which contribute to guessing intelligently refer to two different kinds of clues: linguistic and nonlinguistic.

\section{Using linguistic clues.}

Seeking and using linguistic based clues in order to guess the meaning of what is heard or read in the target language, in the absence of complete knowledge of vocabulary, grammar, or other target language elements. Language-based clues may come from aspects of the target language that the learner already knows, from the learner's own language, or from another language. For instance, if the learner does not know the expression association sans but lucratif ("nonprofit association" in French), 
previous knowledge of certain words in English (association, lucrative) and French (sans = without) could give clues to the meaning of the unknown word, but (aim, goal) and of the whole expression.

\section{Using other clues.}

Seeking and using clues that are not language-based in order to guess the meaning of what is heard or read in the target language, in the absence of complete knowledge of vocabulary, grammar, or other target language elements. Nonlanguage clues may come from a wide variety of sources: knowledge of context, situation, text structure, personal relationships, topic, or "general world knowledge." For example, if the learner does not know what is meant by the words vends or a venre in the French newspaper, noticing that these words are used in the context of classified ads, and that they are followed by a list of items and prices, provides clues suggesting that these terms probably refer to selling.

\section{Overcoming Limitations in Speaking and Writing}

Eight strategies are used for overcoming limitations in speaking and writing. Some of these are dedicated solely to speaking, but some can be used for writing, as well.

\section{Switching to the mother tongue.}

Using the mother tongue for an expression without translating it, as in ich bin eine 'girl'. This strategy may also include adding word endings from the new language onto words from the mother tongue.

\section{Getting help.}

Asking someone for help by hesitating or explicitly asking for the person to provide the missing expression in the target language.

\section{Using mime or gesture}

Using physical motion, such as mime or gesture, in place of an expression to indicate the meaning.

\section{Avoiding communication partially or totally.}

Partially or totally avoiding communication when difficulties are 
anticipated. This strategy may involve avoiding communication in general, avoiding certain topics, avoiding specific expressions, or abandoning communication in mid-utterance.

\section{Selecting the topic.}

Choosing the topic of conversation in order to direct the communication to one's own interests and make sure the topic is one in which the learner has sufficient vocabulary and grammar to converse.

6. Adjusting or approximating the message.

Altering the message by omitting some items of information, making ideas simpler or less precise, or saying something slightly different that means almost the same thing, such as saying pencil for pen.

7 . Coining words.

Make up new words to communicate the desired idea, such as paperholder for notebook.

8. Using a circumlocution or synonym

Getting the meaning across by describing the concept (circumlocution) or using a word that means the same thing (synonym); for example, "what you use to was dishes with" as a description for dishrag.

\section{Metacognitive Strategies}

"Metacognitive" means beyond, beside, or with the cognitive. Therefore, metacognitive strategies are actions which go beyond purely cognitive devices, and which provide a way for learners to coordinate their own learning process.

\section{Centering Your Learning}

This set of three strategies helps learners to converge their attention and energies on certain language tasks, activities, skills or materials. Use of these strategies provides a focus for language learning. 
1 . Overviewing and linking with already known material.

Overviewing comprehensively a key concept, principle or set of materials in an upcoming language activity and associating it with what is already known. This strategy can be accomplished in many different ways, but it is often helpful to follow three steps: learning why the activity is being done, building the needed vocabulary, and making the associations.

\section{Paying attention.}

Deciding in advance to pay attention in general to language learning task and to ignore distractors (by directed attention) and/or to pay attention te specific aspects of the language or to situational details (by selective attention).

3. Delaying speech production to focus on listening.

Deciding in advance to delay speech production in the new language either totally or partially, until listening comprehension skills are better developed. Some language theorists encourage a "silent period" of delayed speech as part of the curriculum, but there is debate as to whether all students require this.

\section{Arranging and Planning Your Learning}

This set contains six strategies, all of which help learners to organize and plan so as to get the most out of language learning. These strategies touch many areas: finding out about language learning, organizing the schedule and the environment, setting goals and objectives, considering task purposes, planning for tasks, and seeking chances to practice the language.

\section{Finding out about language learning.}

Making efforts to find out how language learning works by reading books and talking with other people, and then using this information to help improve one's own language learning.

\section{Organizing.}

\section{Understanding and using conditions related to optimal learning of}


the new language; organizing one's schedule, physical environment (e.g., space, temperature, sound, lighting), and language learning notebook.

\section{Setting goals and objectives.}

Setting aims for language learning, including long-term goals (such as being able to use the language for informal conversation by the end of the year) or short-term objectives (such as finishing reading a short story by Friday).

\section{Identifying the purpose of a language task.}

Deciding the purpose of a particular language task involving listening, reading, speaking, or writing. For example, listening to the radio to get the latest news on the stock exchange, reading a play for enjoyment, speaking to the cashier to buy a train ticket, writing a letter to persuade a friend not to do something rash. (This is sometimes known as Purposeful Listening/Speaking/Reading/Writing.)

\section{Planning for a language task.}

Planning for the language elements and functions necessary for an anticipated language task or situation. This strategy includes four steps: describing the task or situation, determining its requirements, checking one's own linguistic resources, and determining additional language elements or functions necessary for the task or situation.

\section{Seeking practice opportunities.}

Seeking out or creating opportunities to practice the new language in naturalistic situations, such as going to a second/foreign language cinema, attending a party where the language will be spoken, or joining an international social club. Consciously thinking in the new language also provides practice opportunities.

\section{Evaluating Your Leaming}

In this set are two related strategies, both aiding learners in checking their language performance. One strategy involves noticing and learning from errors, and the other concerns evaluating overall progress. 
1. Self-monitoring.

Identifving errors in understanding or producing the new language, determining which ones are important (those that cause serious confusion or offense), tracking the source of important errors, and trying to eliminate such errors.

\section{Self-evaluating.}

Evaluating one's own progress in the new language, for instance, by checking to see whether one is reading faster and understanding more than 1 month or 6 months ago, or whether one is understanding a greater percentage of each conversation.

\section{Affective Strategies}

The term affective refers to emotions, attitudes, motivations, and values. Language learners can gain control over these factors through affective strategies.

Lowering Your Anxiety

Three anxiety-reducing strategies are listed here. Each has a physical component and a mental component.

1. Using progressive relaxation, deep breathing, or meditation

Using the technique of alternately tensing and relaxing all of the major muscle groups in the body, as well as the muscles in the neck and face, in order to relax; or the technique of breathing deeply from the diaphragm; or the technique of meditating by focusing on a mental image or sound.

2. Using music relax.

Listening to soothing music, such as a classical concert, as a way to

3. Using laughter.

Using laughter to relax by watching a funny movie, reading a humorous book, listening to jokes, and so on. 


\section{Encouraging Yourself}

This set of three strategies is often forgotten by language learners, especially those who expect encouragement mainly from other people and do not realize they can provide their own. However, the most potent encouragement -- and the only available encouragement in many independent language learning situations - may come from inside the learner. Self-encouragement includes saying supportive things, prodding oneself to take risks wisely, and providing rewards.

\section{Making positive statements.}

Saying or writing positive statements to oneself in order to feel more confident in learning the new language.

\section{Taking risks wisely.}

Pushing oneself to take risks in a language learning situation, even though there is a chance of making a mistake or looking foolish. Risks must be tempered with good judgment.

\section{Rewarding yourself.}

Giving oneself a valuable reward for a particularly good performance in the new language.

\section{Taking Your Emotional Temperature}

The four strategies in this set help learners to assess their feelings, motivations, and attitudes and, in many cases, to relate them to language tasks. Unless learners know how they are feeling and why they are feeling that way, they are less able to control their affective side. The strategies in this set are particularly helpful for discerning negative attitudes and emotions that impede language learning progress

\section{Listening to your body.}

Paying attention to signals given by the body. These signals may be negative, reflecting stress, tension, worry, fear, and anger; or they may be positive, indicating happiness, interest, calmness, and pleasure. 
2. Using a checklist.

Using a checklist to discover feelings, attitudes, and motivations concerning language learning in general, as well as concerning specific language tasks.

3. Writing a language learning diary

Writing a diary or journal to keep track of events and feelings in the process of learning a new language.

4. Discussing your feelings with someone else.

Talking with another person (teacher, friend, relative) to discover and express feelings about language learning.

\section{Social Strategies}

Language is a form of social behavior, it is communication, and communication occurs between and among people. Learning a language thus involves other people, and appropriate social strategies are very important in this process.

\section{Asking Questions}

This set of strategies involves asking someone, possibly a teacher or native speaker or even a more proficient fellow learner, for clarification, verification, or correction.

\section{Asking for clarification or verification.}

Asking the speaker to repeat, paraphrase, explain, slow down, or give examples; asking if a specific utterance is correct or if a rule fits a particular case; paraphrasing or repeating to get feedback on whether something is correct.

\section{Asking for correction.}

Asking someone for correction in a conversation. This strategy most often occurs in conversation but may also be applied to writing.

This set of two strategies involves interacting with one or more 
people to improve language skills. These strategies are the basis of cooperative language learning, which not only increases learners' language performance but also enhances self-worth and social acceptance.

\section{1 . Cooperating with peers.}

Working with other language learners to improve language skills. This strategy can involve a regular learning partner or a temporary pair or small group. This strategy frequently involves controlling impulses toward competitiveness and rivalry.

2. Cooperating with proficient users of the new language.

Working with native speakers or other proficient users of the new language, usually outside of language classroom. This strategy involves particular attention to the conversational roles each person takes.

\section{Empathizing With Others}

Empathy can be developed more easily when language learners use these two strategies.

\section{Developing cultural understanding.}

Trying to empathize with another person through learning about the culture, and trying to understand the other person's relation to that culture.

2. Becoming aware of others' thoughts and feelings.

Observing the behavior of others as a possible expression of their thoughts and feelings; and when appropriate, asking about thoughts and feelings of others. 
APPENDIX C

THINK-ALOUD PROTOCOL TASKS 


\section{TASK I}

\section{REVIEW OF TENSES}

Directions: Complete the sentences with the words in parentheses. Use any appropriate tense.

Almost every part of the world (1. experience) an earthquake in recent years, and almost every part of the world (2.experience) earthquakes in the years to come. Since the ancient Chinese (3. begin) to keep records thousands of years ago, more than 13 million earthquakes (4. occur) worldwide by some estimates.

What (5.cause) earthquakes? Throughout time, different cultures (6. develop) myths to explain these violent earth movements.

According to a Japanese myth, a playful catfish lives in the mud under the earth. Whenever it feels like playing, it (7. wave) its fat tail around in the mud. The result?

Earthquakes. From India comes a story of six strong elephants who (8. hold) up the earth on their heads. Whenever one elephant (9. move) its head, the earth trembles.

Nowadays, although scientists (10. know) more about the causes of earthquakes, they still can't prevent the terrible damage.

One of the strongest quakes in this century (11. happen) 
in Anchorage, Alaska, on March 24, 1964, at about six

o'clock in the evening. When the earthquake (12. strike)

that evening, many families (13. sit) down to eat

dinner. People in the city (14. find, suddenly)

themselves in the dark because most of the lights in the city went out when the earthquake occurred. Many people (15. die) instantly when tall buildings (16. collapse) and (17. send) tons of bricks and concrete crashing into the streets.

When (18. occur, the next earthquake)

? No one really knows for sure. 
TASK II

\section{MEMORIZATION EXERCISE}

You have five minutes to memorize the words below. After that you will be asked to write down as many of them as you can recall. You may use your paper and pencil to work with them if you want. Be sure to think aloud as you are doing this paper clips rigidity notepad telephone hositile* ribbon floppy disk stapler** serene printer answering machine stapler** tape personality pens

* this is how it was spelled on the task

** these two items appeared twice on the task

\section{TASK III \\ COMPOSITION}

The Traffic Officer (McKay \& Rosenthal, 1980)

You are a traffic officer. As part of your job, you must file a report of accidents you covered while on duty. Yesterday, you were on the scene of auto accident that took place on a country road. You now need to file a report of that accident.

Task: Write a report of the accident. The following information is what you wrote down in your note pad. Use this information to write your report on what happened yesterday. Be certain to make clear the sequence of events.

Time: $\quad$ 7:20 A.M., April 14

Place Highway 652, two miles south of the city 
An overturned Volkswagen on the shoulder of the southbound lane Skid marks leading from the southbound lane to the Volkswagen A pickup truck blocking the northbound lane of traffic Skid marks going from the southbound lane into the northbound lane (leading to the pickup truck)

Front of a Chevrolet station wagon smashed against the side of the pickup truck

(drawing of the traffic accident)

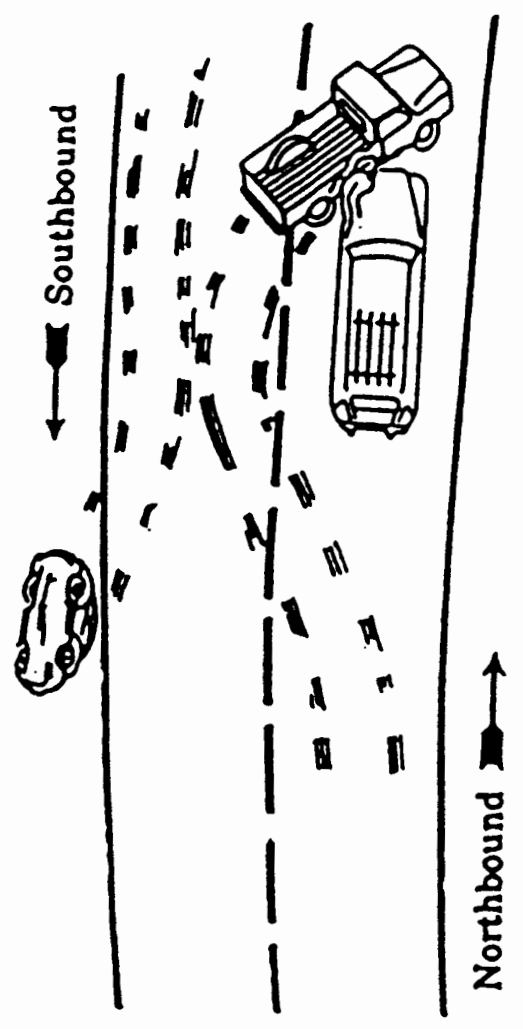

(McKay \& Rosenthal, 1980, p.47) 
APPENDIX D

SAMPLE OF TRANSCRIPTS FROM THINK-ALOUD PROTOCOL 
SAMPLE OF THINK-ALOUD TRANSCRIPT

FROM PART OF COMPOSITION TASK

(LESS PROEICIENT LEARNER)

The following information is what you wrote down on your note pad. Use the information to write your report on what happened yesterday. Be certain to make clear the sequence of events. Huh. So, from this information I imagine what

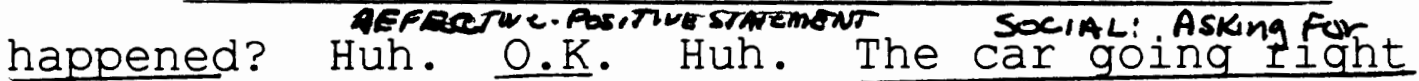
sidde, right? (researcher) Yes, yeah. Cars are in the right hand side in America, left side -Japan. (subject) giggle. Yes, O.K.... So, I think..a pick Place: Highway (researcher)Write it in sentence form. (subject) Social-coopeparing O.K. umm, What's happening? (researcher) un huh (subject) What happened? (researcher) time, place, everything (subject) Everything?

(researcher) un huh (subject) Oh. Ahh..... It it was happened at in, at (researcher) speak a little louder (subject) at Highway $652 \mathrm{umm}$, two miles two miles south of the city uh. in, on April 14. Seven-twenty a.m. When a pick -up truck uhh...umm. pickup truck... What should I social: Asking for Clarification
wite about, how happened? how was the, or after the accident? (researcher) tell what happened, how did it happen (subject) Oh, I guess? 


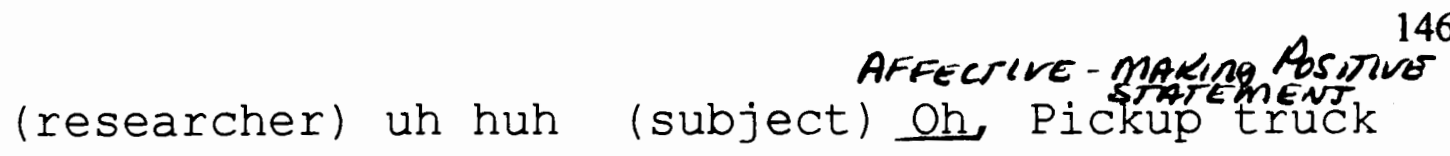
was.. going was going (researcher) speak up (subject) was going too, too uhh.......close? (researcher) What are you thinking? (subject) I AfFeCIUE: Disceus feelings don't know. I cannot remember the words. A pickup truck was going to to (researcher) so say that. I can't find the word. what is the word? AFFETIVE - LAUGHTER (subject) What is the word for for 'giggle'

\section{AFECTIVE.MAKIMS POSITIUE THFOMUNT} hmm... ok A pickup A pickup truck was going COMPENSATION-APproximating MESAGE to..... go....go through the the station wagon.... wagon. When the pickup truck was going to go through the station wagon, from.. uh.... northbound to. from northbound to southbound b-o$u-n-d$. The Volks, the Volkswagen came was com coming front of, of a (researcher) speak-up (subject) a pick-up truck was southbound. The Volkswagen, was, when the pickup truck was (whisper, unintelligible) northbound to southbound the Volkswagen was coming from the (researcher) (unintelligible) (subject) yeah, Umm..umm coming in front of the pick-up truck..so..the, ah, (pick-up truck, pingk-up truck tried tried to tried to tried to hah... tried, tried to uhh).. Es-Cape Irom the Volkswagen, but it was too late. And then uh...uh...... (researcher) speak aloud (subject)pick-up truck smashed sssmmasheded against the side of the sta- 
METACOGNITIVE-SELF-EVACUation/moNiTORIIIT tion wagon. It was happened at Highway 652, two miles south of the city on April 14, seven-twenty a.m. When a pickup truck was going to go through the station wagon from northbound to southbound. The Volks-wagen umm... was coming.. from.. coming from front, coming from, from, front, front, coming front of a pickup truck so a pickup truck tried to escape from the Volkswagen, but it was too late. 
APPENDIX E

STATISTICS FROM SILL DATA 
$\rightarrow$ NPAR TESTS

$\rightarrow \quad / M-W=a b c d e f$ ave $B Y$ learnp $\left(\begin{array}{ll}1 & 0\end{array}\right)$

$\rightarrow \quad / S T A T I S T I C S=$ DESCRIPTIVES QUARTILES

$\rightarrow$ /MISSING ANAIYSIS.

$\begin{array}{lrrrrr} & \mathrm{N} & \text { Mean } & \text { Std Dev } & \text { Minimum } & \text { Maximum } \\ \text { A } & 17 & 3.04706 & .47185 & 2.30 & 4.10 \\ \mathrm{~B} & 17 & 3.54118 & .38739 & 2.90 & 4.40 \\ \mathrm{C} & 17 & 3.56471 & .56672 & 2.80 & 4.70 \\ \mathrm{D} & 17 & 3.94706 & .48877 & 3.30 & 4.80 \\ \mathrm{E} & 17 & 3.14706 & .61757 & 2.30 & 4.50 \\ \text { F } & 17 & 3.78235 & .74686 & 2.50 & 5.00 \\ \text { AVE } & 17 & 3.52941 & .29951 & 3.00 & 4.00 \\ \text { LEARNP } & 17 & .70588 & .46967 & .00 & 1.00 \\ & & & \text { Median) } & & \\ & & 25 t h & 50 t h & 75 t h & 3.3000 \\ \text { A } & \mathrm{N} & \text { Percentile } & \text { Percentile } & \text { Percentile } \\ \text { B } & 17 & 2.7500 & 3.2000 & 3.7500 & 4.1000 \\ \text { C } & 17 & 3.3000 & 3.6000 & 4.3000 \\ \text { D } & 17 & 3.0000 & 3.5000 & 3.5000 \\ \text { E } & 17 & 3.5000 & 3.9000 & 4.4500 & 3.8000\end{array}$

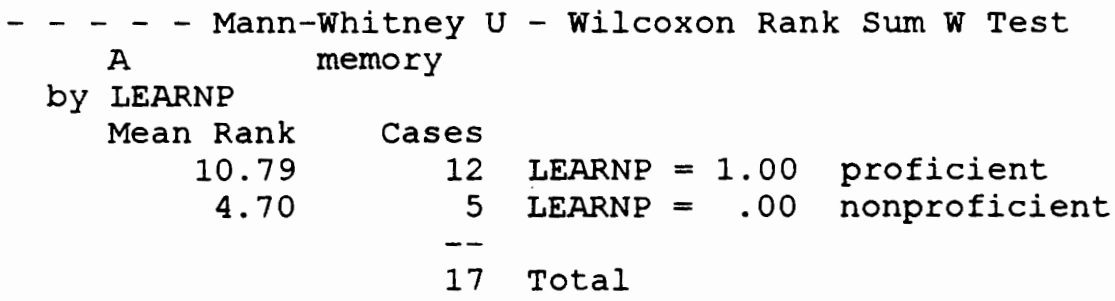

Exact

ties

$$
\mathrm{U}
$$$$
23.0
$$

$$
\text { W }
$$$$
38.0
$$

Corrected for

$$
2 \text { 2-Tailed P }
$$




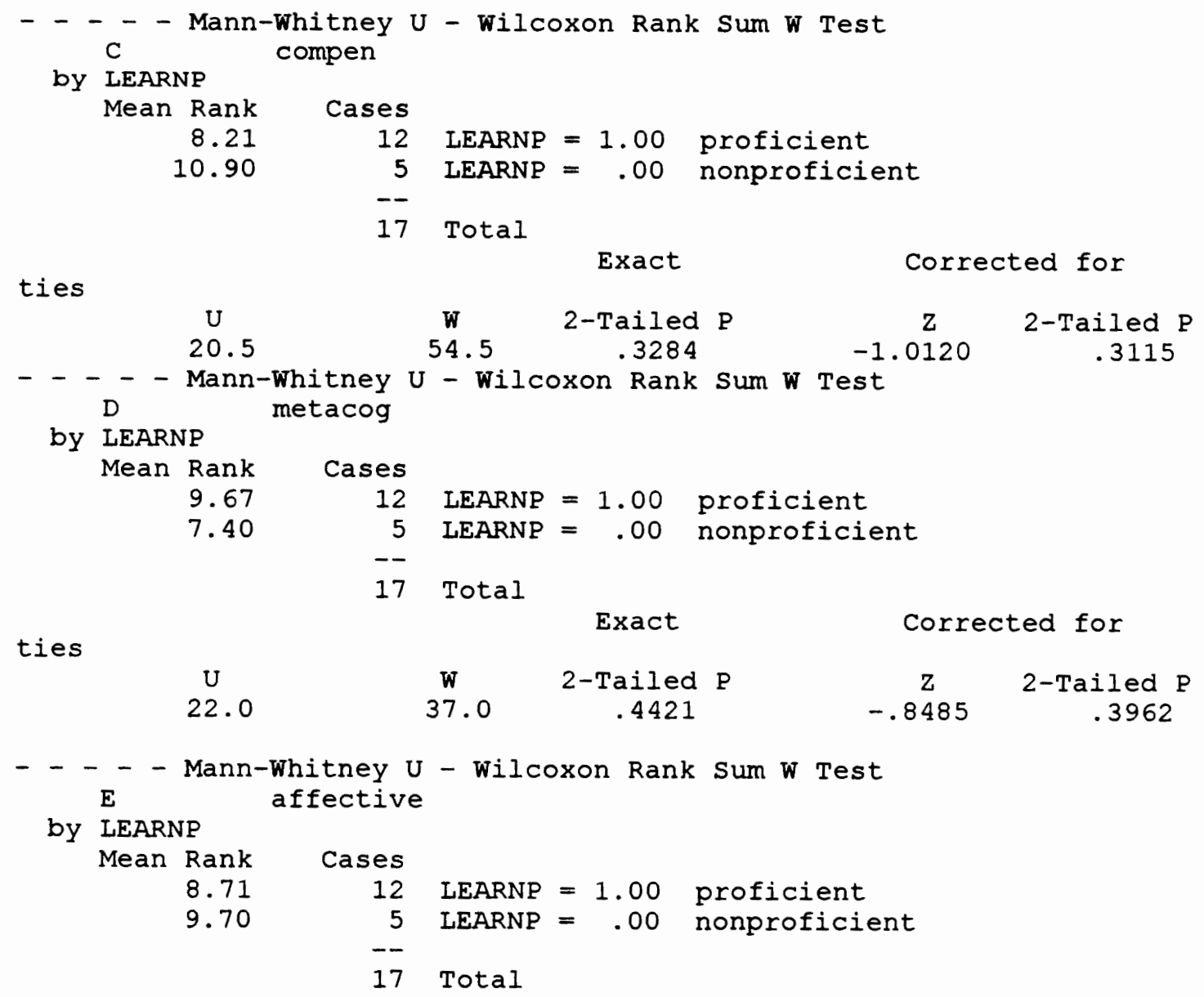

\section{Exact}

$P$

(1)




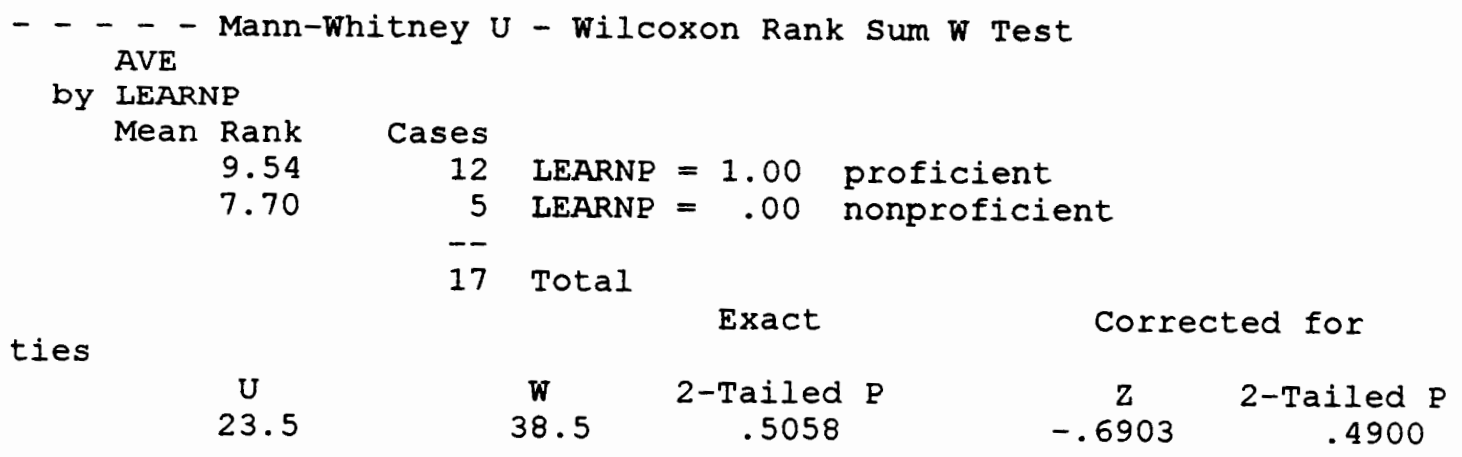




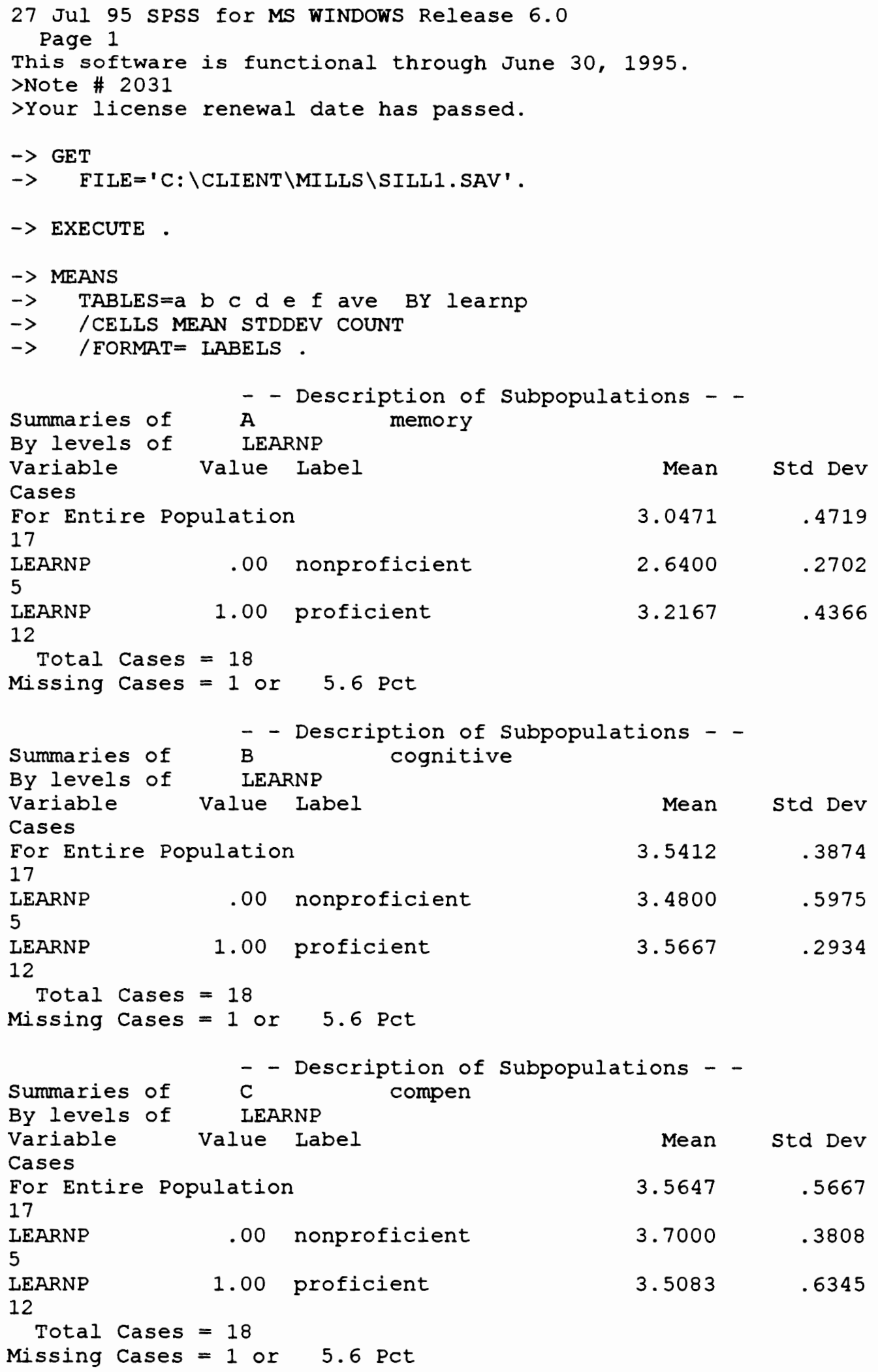




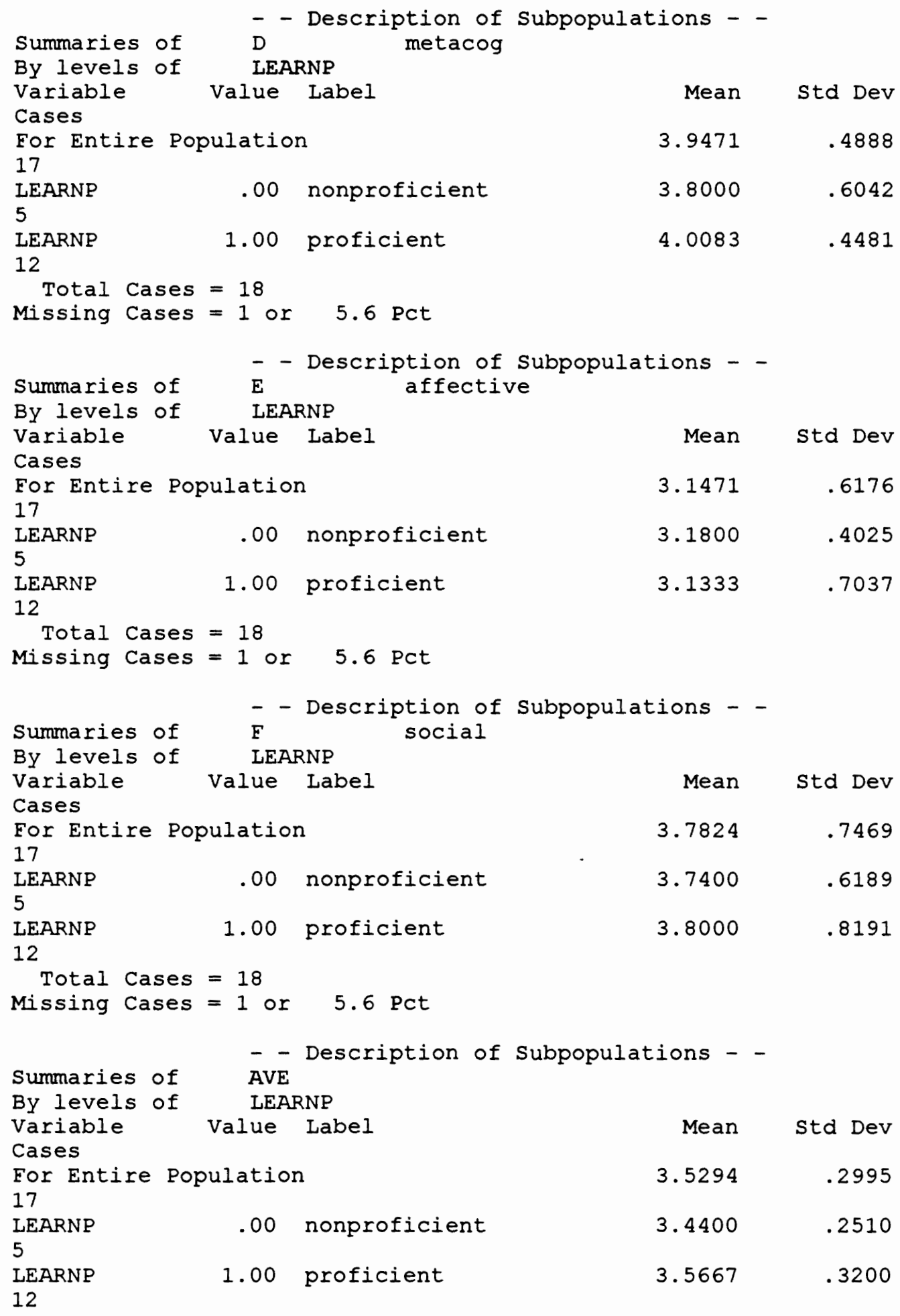


Total Cases $=18$

Missing Cases $=1$ or $5.6 \mathrm{PC}$ 
APPENDIX F

STUDENT DATA FROM SILL AND THINK-ALOUD PROTOCOL 


\section{DATA FROM PART I OF STUDY}

SILL Average Score for Each Strategy and overall SILL Average Teacher Strategies SLL Ranking memory cognitive compen metacog affective social Avg

Students

(Advanced Class)

$\begin{array}{llllllll}1 & 3.4 & 4.1 & 3.0 & 4.7 & 4.5 & 3.8 & 4.0 \\ 2 & 3.6 & 3.8 & 3.8 & 3.9 & 2.3 & 2.7 & 3.4 \\ 3 & 3.3 & 3.6 & 4.7 & 4.4 & 3.3 & 4.4 & 3.9 \\ 4 & 3.2 & 3.4 & 4.2 & 3.6 & 4.2 & 4.7 & 3.9 \\ 5 & 3.3 & 3.3 & 4.0 & 3.3 & 3.2 & 3.5 & 3.4\end{array}$

$\begin{array}{cccccccc}6 & 2.7 & 3.6 & 4.2 & 4.3 & 2.7 & 4.5 & 3.7 \\ 7 & 4.1 & 3.7 & 2.8 & 4.2 & 3.7 & 4.0 & 3.8 \\ 8^{*} & 2.8 & 3.9 & 3.0 & 4.1 & 2.7 & 4.3 & 3.5 \\ 9 & 3.2 & 3.1 & 3.0 & 3.7 & 2.7 & 3.0 & 3.1 \\ 10 & 3.3 & 3.3 & 3.2 & 4.3 & 3.3 & 5.0 & 3.7 \\ 11 & 2.4 & 3.3 & 3.2 & 3.3 & 2.5 & 3.2 & 3.0 \\ 12 & 3.3 & 3.7 & 3.0 & 4.3 & 2.5 & 2.5 & 3.4 \\ 13 & 2.3 & 4.4 & 3.3 & 4.8 & 3.2 & 4.3 & 3.8 \\ 14^{*} & 2.9 & 3.5 & 4.2 & 3.3 & 2.7 & 4.5 & 3.5 \\ 15 & 2.4 & 2.9 & 4.0 & 4.3 & 3.0 & 3.2 & 3.2 \\ 16 & 2.8 & 3.0 & 3.5 & 3.6 & 3.2 & 3.2 & 3.2 \\ 17 & 2.8 & 3.6 & 3.5 & 3.9 & 3.8 & 3.5 & 3.5 *\end{array}$

\# 8 = Proficient Leaner in Think-Aloud Protocol Study

\#14= Less Proficient Learner in Think-Aloud Protocol Study

Compen.$=$ Compensation $\quad$ Metacog.$=$ Metacognitive 
Proficiency Scores for Students

\begin{tabular}{|c|c|c|c|}
\hline Student & MTELP* & CELT* $^{*}$ & $\begin{array}{r}\text { Weighted } \\
\text { Score** }\end{array}$ \\
\hline \multicolumn{4}{|c|}{ (Advanced Students) } \\
\hline$\# 1$ & $81 \%$ & 72 & 78.1 \\
\hline 2 & 83 & 68 & 78.0 \\
\hline 3 & 70 & 76 & 72.0 \\
\hline 4 & 68 & 96 & 77.3 \\
\hline 5 & 67 & 76 & 70.0 \\
\hline \multicolumn{4}{|c|}{ (Upper Intermediate Students) } \\
\hline 6 & 78 & 78 & 82.0 \\
\hline 7 & 77 & 86 & 80.0 \\
\hline 8 & 70 & 92 & 77.3 \\
\hline 9 & 69 & 96 & 77.9 \\
\hline 10 & 70 & 82 & 74.0 \\
\hline 11 & 69 & 84 & 73.9 \\
\hline 12 & 70 & 76 & 72.0 \\
\hline 13 & 58 & 84 & 66.6 \\
\hline 14 & 60 & 80 & 66.6 \\
\hline 15 & 65 & 66 & 65.4 \\
\hline 16 & 57 & 68 & 60.6 \\
\hline 17 & 55 & 62 & 57.4 \\
\hline \multicolumn{4}{|c|}{$\begin{array}{l}\text { * CELT }=\text { Comprehensive English Language Test (listening portion) } \\
\text { Michigan Test of English Language Proficiency, nonsecure version }\end{array}$} \\
\hline
\end{tabular}




\section{DATA FROM PART II OF STUDY}

(Proficient Learner)

Task
SILL

\begin{tabular}{|c|c|c|c|c|c|}
\hline Strategies & $\# 1$ & $\# 2$ & $\# 3$ & total & Score \\
\hline Memory & 0 & 15 & 0 & 15 & 2.8 \\
\hline Cognitive & 20 & 7 & 4 & 31 & 3.9 \\
\hline Compensation & 2 & 0 & 6 & 8 & 3.0 \\
\hline Metacognitive & 3 & 4 & 11 & 18 & 4.1 \\
\hline Affective & 15 & 15 & 38 & 68 & 2.7 \\
\hline Social & 5 & 8 & 11 & 24 & 4.3 \\
\hline Total & 45 & 49 & 70 & 164 & 3.5 \\
\hline
\end{tabular}

(Less Proficient Learner)
SILL

$\begin{array}{lrrrrr}\text { Strategies } & \# 1 & \# 2 & \# 3 & \text { total } & \text { Score } \\ \text { Memory } & 0 & 8 & 0 & 8 & 2.9 \\ \text { Cognitive } & 23 & 18 & 9 & 50 & 3.5 \\ \text { Compensation } & 2 & 0 & 2 & 4 & 4.2 \\ \text { Metacognitive } & 1 & 5 & 7 & 13 & 3.3 \\ \text { Affective } & 2 & 4 & 7 & 13 & 2.7 \\ \text { Social } & 3 & 2 & 6 & 11 & 4.5 \\ \text { Total } & 31 & 37 & 31 & 99 & \text { Average 3.5 }\end{array}$

Task \# 1=Verb Tenses $\quad$ Task \# 2=Memorization

Task \# 3=Composition 